\title{
Similarity solutions to an averaged model for superconducting vortex motion
}

\author{
G. RICHARDSON \\ Section of Theoretical Mechanics, University of Nottingham, Nottingham NG7 2RD, UK
}

(Received 27 March 2002; revised 27 May 2003)

Under certain conditions the motion of superconducting vortices is primarily governed by an instability. We consider an averaged model, for this phenomenon, describing the motion of large numbers of such vortices. The model equations are parabolic, and, in one spatial dimension $x$, take the form

$$
\begin{aligned}
& H_{2 t}=\frac{\partial}{\partial x}\left(\left|H_{3} H_{2 x}-H_{2} H_{3 x}\right| H_{2 x}\right), \\
& H_{3 t}=\frac{\partial}{\partial x}\left(\left|H_{3} H_{2 x}-H_{2} H_{3 x}\right| H_{3 x}\right) .
\end{aligned}
$$

where $\mathrm{H}_{2}$ and $\mathrm{H}_{3}$ are components of the magnetic field in the $y$ and $z$ directions respectively. These equations have an extremely rich group of symmetries and a correspondingly large class of similarity reductions. In this work, we look for non-trivial steady solutions to the model, deduce their stability and use a numerical method to calculate time-dependent solutions. We then apply Lie Group based similarity methods to calculate a complete catalogue of the model's similarity reductions and use this to investigate a number of its physically important similarity solutions. These describe the short time response of the superconductor as a current or magnetic field is switched on (or off).

\section{Introduction}

So-called superconducting materials, when cooled through a critical temperature, make a transition to the superconducting state in which they have the ability to conduct electric currents without resistance. A less well-known property exhibited by such materials when in this state, termed the Meissner effect, is a strong propensity to exclude magnetic fields. There is however a limit to a material's capacity to do this and large fields, exceeding the so-called critical field, act to destroy its superconducting properties. For fields not exceeding, but sufficiently close to, the critical field this, combined with the Meissner effect, leads to some interesting behaviour. In so-called Type-I superconductors the field partially penetrates the material through normal regions where it exceeds the critical field. These are then separated from the superconducting regions, in which the field is extremely small, by a sheet-like phase boundary. In Type-II superconductors, however, the field penetrates along a number of separate filaments, each associated with one quantum of magnetic flux. Around these filaments, or vortices, the superconducting properties are partially destroyed. This state, in which the magnetic field penetrates throughout the 
region along vortices, has been termed the mixed state because there is no large-scale separation of normal and superconducting phases as in a Type-I superconductor.

Many models have been proposed to describe the behaviour of superconductors. One of the most successful of these is the so-called Ginzburg-Landau model [17], which describes the behaviour in terms of two variables, the magnetic field $\boldsymbol{H}$ and an order parameter $\psi$, the modulus of which is related to the local density of superconducting electrons. Reviews of the mathematical aspects of this model are given in Chapman et al. [10] and Du et al. [13]. Its most notable triumph was the prediction of vortices by Abrikosov [1]. An important feature of the model is that the morphology of solutions is found to be crucially dependent on a certain material parameter, the Ginzburg-Landau parameter $\kappa$, which gives the ratio of typical variations in the magnetic field (the penetration depth $\lambda$ ) to typical variations in the order parameter (the coherence length $\xi$ ). In particular, the value of $\kappa$ determines whether Type-I $(\kappa<1 / \sqrt{2})$ or Type-II $(\kappa>1 / \sqrt{2})$ behaviour is exhibited. Here we shall be interested in the high- $\kappa$ limit in which the thickness of the core of the vortex, outside which the size of the order parameter $\psi$ does not vary appreciably, tends to zero. For a large number of materials, typically alloys, this limit provides a good description, examples being $\mathrm{NbSn}, \kappa=15.3$; $\mathrm{NbNi}, \kappa=28.0$ and $\mathrm{V}_{3} \mathrm{G}, \kappa=25.3$.

The macroscopic response of a Type-II superconductor to changes in the surrounding magnetic fields is primarily governed by the motion of large numbers of superconducting vortices. Furthermore vortex motion within the superconductor leads to energy dissipation which in many applications is undesirable. Vortex motion may be retarded by introducing impurities into a superconducting material; these act to trap vortices and this effect is termed pinning (see, for example, Chapman [7]). So-called critical state (stick-slip) models are widely accepted as providing a good description of the long-timescale behaviour for vortex motion in two-dimensional geometries (see Bean [2] and Chapman [7]). However, there remain unresolved issues in the extension of these models to fully-three dimensional geometries with several candidate models in existence $[12,5,7]$. In this work, we shall be interested in vortex motion in the absence of pinning, but note that the physics of vortex flow in pinned superconductors is essentially the same as in un-pinned materials and so our work has potential applications to devices such as fault-current limiters in which the pinning potential is exceeded and an unretarded vortex flow occurs. Furthermore the correct formulation of a three-dimensional critical state model (for pinned superconductors) requires that the short-time scale flow of vortices is properly understood, and in this respect, we expect this work will further understanding.

Vortices move in response to their curvature and gradients in the magnetic field (electric currents). In the limit as $\kappa$ becomes large, and in the absence of vortex pinning, the vortices move with velocity $\boldsymbol{v}$ where

$$
\frac{\beta}{2} \boldsymbol{v}=\frac{C \log \kappa}{2} \boldsymbol{n}+(\nabla \wedge \boldsymbol{H}) \wedge \boldsymbol{t}
$$

This formula is systematically derived $[8,14]$ from the underlying Ginzburg-Landau model [17]. Here the term $\beta / 2$ is a mobility coefficient, $C$ the curvature of the vortex line, $\boldsymbol{H}$ the regular part of the magnetic field evaluated along the vortex line, $\boldsymbol{t}$ its tangent and $\boldsymbol{n}$ its principal normal. The first term in (1.1) is a line tension whose action is to shorten the vortex curve while the second is a driving force arising from action of the electric 
current on the vortex - this is often termed the motion due to the Lorenz force. The problem for vortex motion is coupled to a problem for the magnetic field $\boldsymbol{H}$,

$$
\nabla \wedge(\nabla \wedge \boldsymbol{H})+\boldsymbol{H}=2 \pi \sum_{k=1}^{n} \boldsymbol{\delta}_{\Gamma_{k}}(\boldsymbol{x}),
$$

where the summation is over all the vortices present in the superconductor, $\Gamma_{k}$ is the $k$ th vortex curve and

$$
\boldsymbol{\delta}_{\Gamma_{k}}(\boldsymbol{x})=\int_{\Gamma_{k}} \delta(x-\hat{x}) \delta(y-\hat{y}) \delta(z-\hat{z}) d \hat{\boldsymbol{x}} .
$$

In practice, it is not feasible to use this model directly to simulate the response of a macroscopic superconducting sample because of the vast number of vortices typically found inside such samples. This has led to the search for an averaged model of superconducting motion which is capable of giving an approximate description of the motion of large numbers of vortices. Modelling of this form was first conducted by Brandt [5] on the basis of heuristic arguments. A subsequent formal derivation of an averaged model from (1.1)-(1.2) was made by Chapman [6] in a regime where vortices are sufficiently densely packed, and the magnetic fields correspondingly large, so that the the first term on the right of (1.1) can be neglected. In certain special geometries, such as thin films, this turns out to be a reasonable assumption however in most three-dimensional situations such an assumption leads to a model which is linearly ill-posed [20]. The reason for this pathological behaviour is a three-dimensional vortex instability that occurs wherever there is a component of $\nabla \wedge \boldsymbol{H}$ in the direction of the vortex tangent $\boldsymbol{t}$; this instability was first noted by Clem [11]. Over a short time scale, the instability causes vortex lines to develop a highly curved spiral structure which invalidates the assumption that the second term on the right-hand side of (1.1) is much larger than the first. A more detailed investigation, carried out in earlier work [18], reveals that the instability grows so as to try to align the vortex tangent perpendicular to the local electric current density $\boldsymbol{j}=\nabla \wedge \boldsymbol{H}$ or, taking an alternative viewpoint, that the resulting vortex motion causes an electric field parallel to the current density $\boldsymbol{j}$.

The implications of the evolution of this instability has been considered by Chapman \& Richardson [9], who proposed a model for the evolution of large numbers of vortices in a superconductor without pinning. For more details on the derivation and the regime of validity we refer the reader to Chapman [7]. Here we shall be content with writing down the model and some brief explanation. The long wave limit of the model (see equations (5.9)-(5.11) of Chapman [7]), which describes the evolution over lengthscales much greater than the penetration depth of the material (typically about $10^{-7} \mathrm{~m}$ ) is, in dimensionless variables,

$$
\begin{aligned}
\nabla \cdot \boldsymbol{H} & =0, \\
\boldsymbol{H}_{t}+\nabla \wedge \boldsymbol{E} & =\mathbf{0}, \\
\boldsymbol{j} & =\nabla \wedge \boldsymbol{H} \\
\boldsymbol{E} & =\delta \boldsymbol{H} \wedge\left(\boldsymbol{j} \wedge \frac{\boldsymbol{H}}{|\boldsymbol{H}|}\right)+|\boldsymbol{j} \cdot \boldsymbol{H}| \boldsymbol{j} .
\end{aligned}
$$

Here the first term in the expression for $\boldsymbol{E}$ represents the contribution to the electric 
field from the Lorenz force, while the second represents the contribution from the vortex instability and $\delta$ is the ratio between these two contributions. In the regime of validity of this model (the vortex fluid regime) $\delta$ is small. ${ }^{1}$ In addition to modelling clean superconductors (which have no vortex pinning sites) this model also has applications to vortex flow in superconductors with pinning since, once the pinning potential has been overcome, the flow of vortices obeys the same physics as that for a clean superconductor. It can thus be used to describe vortex flow in devices, such as a fault current limiter, which are designed to give significant flows under certain conditions.

This is the first analysis of this model and, in order to make progress, we limit our investigation to the simplest geometry in which non-trivial solutions to the model can be found. This is the one-dimensional slab $\alpha<x<\beta$ which models a superconducting sheet, or tape. Furthermore, we assume this slab is subjected to a parallel applied magnetic field $\boldsymbol{H}_{\text {appl }}$ and carries a transport current $\boldsymbol{I}_{\text {trans }}$ per unit length which flows in the $y-z$ direction. With this in mind we look for solutions of the form

$$
\boldsymbol{H}=\left(0, H_{2}(x, t), H_{3}(x, t)\right)
$$

and on substitution into (1.3)-(1.6), we find

$$
\begin{aligned}
& H_{2 t}=\frac{\partial}{\partial x}\left(\left|H_{3} H_{2 x}-H_{2} H_{3 x}\right| H_{2 x}\right)+\delta \frac{\partial}{\partial x}\left(H_{2} \frac{\left(H_{2} H_{2 x}+H_{3} H_{3 x}\right)}{\left(H_{2}^{2}+H_{3}^{2}\right)^{1 / 2}}\right), \\
& \left.H_{3 t}=\frac{\partial}{\partial x}\left(\left|H_{3} H_{2 x}-H_{2} H_{3 x}\right| H_{3 x}\right)\right)+\delta \frac{\partial}{\partial x}\left(H_{3} \frac{\left(H_{2} H_{2 x}+H_{3} H_{3 x}\right)}{\left(H_{2}^{2}+H_{3}^{2}\right)^{1 / 2}}\right) .
\end{aligned}
$$

Since $\delta$ is small, we neglect terms involving $\delta$ and solve instead the simplified system

$$
\begin{aligned}
& H_{2 t}=\frac{\partial}{\partial x}\left(\left|H_{3} H_{2 x}-H_{2} H_{3 x}\right| H_{2 x}\right), \\
& H_{3 t}=\frac{\partial}{\partial x}\left(\left|H_{3} H_{2 x}-H_{2} H_{3 x}\right| H_{3 x}\right) .
\end{aligned}
$$

The resulting equations form a parabolic system. Boundary data of the form

$$
\left(0, H_{2}, H_{3}\right)=\boldsymbol{H}_{-} \quad \text { on } x=\alpha, \quad\left(0, H_{2}, H_{3}\right)=\boldsymbol{H}_{+} \quad \text { on } x=\beta,
$$

must be supplied together with initial conditions for $H_{2}$ and $H_{3}$. The boundary data for this problem comes from specifying the applied magnetic field $\boldsymbol{H}_{\text {appl }}$ and the transport current $\boldsymbol{I}_{\text {trans }}$, and is found from the relations

$$
\boldsymbol{H}^{+}+\boldsymbol{H}^{-}=2 \boldsymbol{H}_{\text {appl }}, \quad \boldsymbol{H}^{+}-\boldsymbol{H}^{-}=\left(0, \boldsymbol{I}_{\text {trans }} \cdot \boldsymbol{e}_{z},-\boldsymbol{I}_{\text {trans }} . \boldsymbol{e}_{\boldsymbol{y}}\right) .
$$

Initial conditions are obtained from a knowledge of the initial density of vortices (magnetic field is proportional to vortex density). This model has a high degree of symmetry and a correspondingly large number of similarity reductions. It is, for instance, invariant under

1 In fact rather than being a constant, $\delta$ should be a decreasing function of $|\boldsymbol{j} \cdot \boldsymbol{H}|$, since the Lorenz force has a smaller effect on a vortex with a helical twist than it does on a straight vortex. 
translations in $x$ and in $t$, under rotations of the $\left(H_{2}, H_{3}\right)$ coordinate axes and under the following rescaling:

$$
H_{2} \rightarrow q H_{2}, \quad H_{3} \rightarrow r H_{3}, \quad x \rightarrow p x, \quad t \rightarrow \frac{p^{3}}{q r} t,
$$

where $q, r$ and $p$ are all arbitrary constants. As a result we expect the model to exhibit a large number of similarity reductions.

The aim of the present work is threefold: first, to investigate the steady states to this novel model of supercondcuting vortex motion; secondly, to examine its unsteady behaviour using a numerical scheme; and finally, to derive its similarity reductions and analyse some of its physically pertinent similarity solutions. We now briefly summarise the main results of the first part of the paper. Of particular importance is a steady solution of the form

$$
H_{2}=a x+b, \quad H_{3}=c x+d,
$$

where $a, b, c$ and $d$ are arbitrary constants, which describes a state with uniform current density across the width of the superconductor. It is noteworthy that there is exactly one such steady solution for each set of boundary data (1.11) applied to (1.9)-(1.10). We demonstrate that such solutions are stable to small one-dimensional perturbations and this motivates the search for similarity solutions which describe the evolution from one steady state of the form (1.12) to another such steady state as either, or both, of the applied magnetic field $\boldsymbol{H}_{\text {appl }}$ and the transport current $\boldsymbol{I}_{\text {trans }}$ are impulsively changed. These similarity solutions take one of two forms: the first describes the evolutions from an initially constant magnetic field; while the second describes the evolution from a magnetic field of the type $H_{2}=a x+b, H_{3}=c x+d$ but assumes the impulsively changed boundary magnetic field lies in a particular direction. Both types of solution describe the evolution in a semi-infinite slab $(\beta \rightarrow \infty)$ and thus provide the short time behaviour for the evolution in the finite slab. The long-term behaviour of this evolution is provided by the linear stability analysis. There is also another non-trivial steady solution

$$
H_{3}=k H_{2}(x)
$$

where $k$ is an arbitrary constant. However this solution is of less physical interest because (i) it can only ever be realised if $\boldsymbol{I}_{\text {trans }} \cdot \boldsymbol{H}_{\text {appl }}=0$ and (ii) because, unlike (1.12), neglecting the $O(\delta)$ terms in the full problem (1.7)-(1.8) represents a singular perturbation. The preceding facts leads us to conjecture that solutions to (1.9)-(1.10) satisfying steady boundary conditions which have the property $\boldsymbol{I}_{\text {trans }} \cdot \boldsymbol{H}_{\text {appl }}=\mathrm{H}_{3}^{-} \mathrm{H}_{2}^{+}-\mathrm{H}_{2}^{-} \mathrm{H}_{3}^{+} \neq 0$ decay to the unique steady solution of the form (1.12) which satisfies these boundary conditions.

In $\S 2$ we derive all the steady solutions to (1.9)-(1.10); these fall into two classes, the first being of type (1.12). We then investigate the stability of (1.12) to small onedimensional perturbations before proceeding to look at numerical solutions to the models in $\S 3$. In $\S 4$ we derive the infinitesimal Lie point symmetries of the model. We then use these and the method of invariant surfaces to construct a complete catalogue of the model's similarity reductions. In $\S 5$ we look for travelling wave solutions. Then in $\S 6$ and $\S 7$ we investigate similarity solutions which exhibit decay to steady solutions of the from $H_{2}=b, H_{3}=d$ and $H_{2}=a x+b, H_{3}=c x+d$ respectively. In the penultimate section, we 
investigate a subclass of similarity solutions which can decay to steady solutions of the second kind (1.13), and finally draw conclusions.

\section{Steady solutions}

Looking for steady solutions to (1.9)-(1.10) by substituting $H_{2}=H_{2}(x)$ and $H_{3}=H_{3}(x)$ results in a set of O.D.E.s that we can integrate exactly. Doing so we find that $\mathrm{H}_{2}$ and $\mathrm{H}_{3}$ either take the form

$$
H_{2}=a x+b, \quad H_{3}=c x+d,
$$

where $a, b, c$, and $d$ are arbitrary constants, or take the form

$$
H_{3}=k H_{2}(x)
$$

where $k$ is an arbitrary constant and $\mathrm{H}_{2}$ an arbitrary function of $x$. Physically, (2.2) corresponds to a solution for which $\boldsymbol{j} \cdot \boldsymbol{H}=\left|H_{3} H_{2 x}-H_{2} H_{3 x}\right| \equiv 0$, whereas solutions of the form (2.1) do not have this property. Thus neglecting the $O(\delta)$ terms in the full system of PDEs (1.7)-(1.8) represents a non-singular perturbation when the steady solution is of the form (2.1), and a singular perturbation when it has the form (2.2). In other words if we include the $O(\delta)$ terms in (1.7)-(1.8) we find an $O(\delta)$ correction to (2.1), whereas we cannot find such a correction to (2.2). In order to work out what happens to such steady solutions to the model (1.9)-(1.10) when we reintroduce the $O(\delta)$, terms we look for solutions to (1.7)-(1.8) of the form

$$
H_{2}=H_{2}(x, \tau), \quad H_{3}=k H_{2}(x, \tau), \quad \tau=\delta t .
$$

Substitution into (1.7)-(1.8) leads to the following slow timescale equation for $\mathrm{H}_{2}$ :

$$
\frac{\partial H_{2}}{\partial \tau}=\left(k^{2}+1\right)^{1 / 2} \frac{\partial}{\partial x}\left(\left|H_{2}\right| \frac{\partial H_{2}}{\partial x}\right) .
$$

This is a well known version of the porous medium equation and implies that over long times such solutions evolve towards a profile of the form $H_{2}= \pm(c x+d)^{1 / 2}$, where $c$ and $d$ are arbitrary constants.

To summarise, if we consider solving for a steady solution in a slab $\alpha<x<\beta$ and imposing the boundary conditions $\boldsymbol{H}=\boldsymbol{H}^{-}$on $x=\alpha$ and $\boldsymbol{H}=\boldsymbol{H}^{+}$on $x=\beta$, then where $\boldsymbol{H}^{-}$and $\boldsymbol{H}^{+}$have the property $\boldsymbol{I}_{\text {trans }} \cdot \boldsymbol{H}_{\text {appl }}=H_{3}^{+} H_{2}^{-}-H_{3}^{-} H_{2}^{+}=0$, there is an infinite set of possible solutions given by (2.2). However, where this condition is not satisfied there is a unique solution of the form (2.1).

\subsection{Linear stability analysis}

We now investigate the stability of the steady solution (2.1) by perturbing about it in the following manner:

$$
\begin{aligned}
& H_{2}=a x+b+\epsilon e^{\sigma t} p(x), \\
& H_{3}=c x+d+\epsilon e^{\sigma t} q(x) .
\end{aligned}
$$


We assume that $H_{2}$ and $H_{3}$ are specified on the boundaries of the body, $x=\alpha$ and $x=\beta$, such that

$$
p(\alpha)=p(\beta)=q(\alpha)=q(\beta)=0 .
$$

Substituting (2.4) into (1.9)-(1.10) leads, after some manipulation, to the following eigenvalue problem:

$$
\begin{gathered}
p^{\prime \prime}-\frac{\sigma}{2(a d-b c)|a d-b c|}\left[(a d-2 b c-a c x) p+\left(a b+a^{2} x\right) q\right]=0, \\
q^{\prime \prime}-\frac{\sigma}{2(a d-b c)|a d-b c|}\left[-\left(c d+c^{2} x\right) p+(2 a d-c b+a c x) q\right]=0,
\end{gathered}
$$

which is supplemented by the boundary conditions (2.5). Note that if $a d=b c$ then we effectively have a solution of the type (2.2) and the preceding long timescale analysis applies so that we need not consider this possibility further. Multiplying (2.6) by $c$ and subtracting (2.7) multiplied by $a$ leads to

$$
\Theta^{\prime \prime}-\frac{\sigma}{|a d-b c|} \Theta=0, \quad \text { where } \Theta=c p-a q .
$$

Multiplying this equation by $\Theta$, integrating from $x=\alpha$ to $x=\beta$ and noting that, since $p$ and $q$ are zero on these boundaries, $\Theta(\alpha)=\Theta(\beta)=0$, we find

$$
\int_{\alpha}^{\beta}\left(\Theta^{\prime 2}+\frac{\sigma}{|a d-b c|} \Theta^{2}\right) d x=0
$$

From this it follows that either $\sigma<0$ or that $\Theta=0$ i.e. $c p(x)=a q(x)$. In the latter instance it can be shown that both (2.6) and (2.7) reduce to

$$
p^{\prime \prime}-\frac{\sigma}{2|a d-b c|} p=0
$$

and we can use a similar argument to that employed above to show that, for a non-trivial solution, we require $\sigma<0$. It follows that the solution (2.1) is stable to one-dimensional perturbations. In light of this, the fact that this steady solution is unique (for a given set of boundary data) and that the equations are parabolic, we make the conjecture that where steady boundary conditions $\left.\boldsymbol{H}\right|_{x=\alpha}=\boldsymbol{H}^{-},\left.\boldsymbol{H}\right|_{x=\beta}=\boldsymbol{H}^{+}$are applied on the edge of the domain, which satisfy the condition $H_{3}^{+} \mathrm{H}_{2}^{-}-\mathrm{H}_{3}^{-} \mathrm{H}_{2}^{+} \neq 0$, the solution tends to the unique steady state of the form (2.1); that is we postulate

$$
\left(H_{2}, H_{3}\right) \rightarrow\left(H_{2}^{-}+\frac{\left(H_{2}^{+}-H_{2}^{-}\right)(x-\alpha)}{(\beta-\alpha)}, H_{3}^{-}+\frac{\left(H_{3}^{+}-H_{3}^{-}\right)(x-\alpha)}{(\beta-\alpha)}\right), \quad \text { as } t \rightarrow \infty .
$$

Remark In the case where the boundary conditions have the property $\mathrm{H}_{3}^{+} \mathrm{H}_{2}^{-}-\mathrm{H}_{3}^{-} \mathrm{H}_{2}^{+}=0$, we can write $H_{3}^{+}-k H_{2}^{+}=H_{3}^{-}-k H_{2}^{-}=0$ for some $k$. We write $W=H_{3}-k H_{2}$, so that $W(\alpha)=W(\beta)=0$, and note that $W$ satisfies the following equation:

$$
W_{t}=\left(D W_{x}\right)_{x} \quad \text { where } \quad D=\left|H_{3} H_{2 x}-H_{2} H_{3 x}\right| .
$$


Multiplying by $W$ and integrating by parts over the interval $(\alpha, \beta)$ we find

$$
\frac{\partial}{\partial t}\left(\int_{\alpha}^{\beta} \frac{W^{2}}{2} d x\right)=-\int_{\alpha}^{\beta} D W_{x}^{2} d x .
$$

It follows that either $D \rightarrow 0$ or $W \rightarrow 0$ as $t \rightarrow \infty$. In either case, this means that the solution approaches a steady solution of the form $H_{3}=k H_{2}(x)$ for large time, although we cannot say what functional form $H_{2}(x)$ takes. $^{2}$

\section{Numerical solution of the model}

We choose to solve equations (1.9)-(1.10) numerically using a semi-implicit finite difference method. As always the reason for choosing to make the scheme, at least partially implicit, is that the stability properties of the resulting difference equations are much improved in comparison to an explicit method and allow the use of relatively large time steps. The implementation of the numerical scheme is not, however, completely standard because we have to allow for the possibility that the diffusion coefficient $\left|\mathrm{H}_{3} \mathrm{H}_{2 x}-\mathrm{H}_{2} \mathrm{H}_{3 x}\right|$ goes to zero at a number of points (where $\mathrm{H}_{3} \mathrm{H}_{2 x}-\mathrm{H}_{2} \mathrm{H}_{3 x}$ passes through zero). In order to overcome this difficulty we introduce the following, physically sensible, regularisation of the diffusion coefficient by writing

$$
\left|H_{3} H_{2 x}-H_{2} H_{3 x}\right| \approx\left(\left(H_{3} H_{2 x}-H_{2} H_{3 x}\right)^{2}+\epsilon\right)^{1 / 2}, \quad \text { where } 0<\epsilon \ll 1 .
$$

This type of approach has been adopted elsewhere to overcome similar difficulties (see, for example, Bowen \& Witelski [4]) and, on substitution of this approximation into the governing equations (1.9)-(1.10), we obtain the system

$$
\begin{aligned}
H_{2 t}= & H_{2 x x} \frac{\left(\left(H_{3} H_{2 x}-H_{2} H_{3 x}\right)\left(2 H_{3} H_{2 x}-H_{2} H_{3 x}\right)+\epsilon\right)}{\left(\left(H_{3} H_{2 x}-H_{2} H_{3 x}\right)^{2}+\epsilon\right)^{1 / 2}} \\
& -H_{3 x x} \frac{H_{2} H_{2 x}\left(H_{3} H_{2 x}-H_{2} H_{3 x}\right)}{\left(\left(H_{3} H_{2 x}-H_{2} H_{3 x}\right)^{2}+\epsilon\right)^{1 / 2}}, \\
H_{3 t}= & H_{3 x x} \frac{\left(\left(H_{3} H_{2 x}-H_{2} H_{3 x}\right)\left(H_{3} H_{2 x}-2 H_{2} H_{3 x}\right)+\epsilon\right)}{\left(\left(H_{3} H_{2 x}-H_{2} H_{3 x}\right)^{2}+\epsilon\right)^{1 / 2}} \\
& +H_{2 x x} \frac{H_{3} H_{3 x}\left(H_{3} H_{2 x}-H_{2} H_{3 x}\right)}{\left(\left(H_{3} H_{2 x}-H_{2} H_{3 x}\right)^{2}+\epsilon\right)^{1 / 2}} .
\end{aligned}
$$

These equations can be discretised in a standard manner by taking the second spatial derivatives of $\mathrm{H}_{2}$ and $\mathrm{H}_{3}$ at the $n+1$ th time step (i.e. the new time where $\mathrm{H}_{2}$ and $\mathrm{H}_{3}$ are still to be evaluated) and all other expressions involving $H_{2}, H_{3}, H_{2 x}$, and $H_{3 x}$ at the $n$ 'th time step (i.e. the old time where $\mathrm{H}_{2}$ and $\mathrm{H}_{3}$ are known). The resulting implicit scheme has good stability properties. The small parameter $\epsilon$ is chosen depending on the initial conditions used; where it is expected that $\mathrm{H}_{3} \mathrm{H}_{2 x}-\mathrm{H}_{2} \mathrm{H}_{3 x}$ does not go through zero we choose $\epsilon=0$, otherwise we choose $\epsilon=0.05$. We note that if we choose $\epsilon$ too

2 We are grateful to an anonymous referee for pointing out this argument. 
small, or correspondingly the mesh size too large, an instability develops about points where $\mathrm{H}_{3} \mathrm{H}_{2 x}-\mathrm{H}_{2} \mathrm{H}_{3 x}=0$. The use of this regularisation is supported by a favourable comparisons between numerically calculated solutions (using appropriate initial data) and a similarity solution displaying a singularity at which $\mathrm{H}_{3} \mathrm{H}_{2 x}-\mathrm{H}_{2} \mathrm{H}_{3 x}=\mathrm{O}$ (see Figures 2 and $6 \mathrm{~b})$.

Figure 1 shows the results of three numerical calculations of the solution to (1.9)-(1.10). In Figure 1(a), the initial conditions $\left(H_{2}, H_{3}\right)=(1+x, 1)$ are used and at time $t=0$ the boundary magnetic fields are switched to $\left.\left(H_{2}, H_{3}\right)\right|_{x=0}=(0,1),\left.\left(H_{2}, H_{3}\right)\right|_{x=2}=(1,-1)$. In the resulting evolution, the diffusion never goes to zero and the solutions are thus non-singular. This is essentially because the end state the solution approaches for large time $\left(H_{2}=x / 2, H_{3}=1-x, H_{3} H_{2 x}-H_{2} H_{3 x}=1 / 2\right)$ has the same sign for $H_{3} H_{2 x}-H_{2} H_{3 x}$ as the initial state. In contrast, in Figure $1(\mathrm{~b})$, the initial conditions $\left(H_{2}, H_{3}\right)=(-2,1+2 x)$ take a different sign for $\mathrm{H}_{3} \mathrm{H}_{2 x}-\mathrm{H}_{2} \mathrm{H}_{3 x}$ than the state the solution approaches for long times $\left(H_{2}, H_{3}\right)=(2-x, 1+2 x)$. Hence the solution displays a singularity at which $H_{3} H_{2 x}-H_{2} H_{3 x}=0$. In Figure 1(c) an impulsive change is made to an initially constant magnetic field of $\left(H_{2}, H_{3}\right)=(2,1)$. Initially, two free boundaries propagate in from each boundary (most clearly seen in the plot of $\mathrm{H}_{3} \mathrm{H}_{2 x}-\mathrm{H}_{2} \mathrm{H}_{3 x}$ ). These eventually meet in the middle of the sample, where they form a singularity with $\mathrm{H}_{3} \mathrm{H}_{2 x}-\mathrm{H}_{2} \mathrm{H}_{3 x}$ taking different signs on either side of it. For long times (longer than is shown in the plots) all three solutions tend towards a unique steady state of the form $H_{2}=a x+b, H_{3}=c x+d$ satisfying the boundary data imposed on the edge of the sample. This seems to be typical behaviour for such solutions and backs up the conjecture made in (2.8).

\section{A complete catalogue of similarity reductions}

We apply the usual Lie group method for determining the classical point symmetries of (1.9)-(1.10) (see for example Bluman and Cole [3]), in the first instance by determining the infinitesimal transformations of the form

$$
\begin{aligned}
t^{*} & \sim t+\varepsilon T\left(H_{2}, H_{3}, x, t\right), \\
x^{*} & \sim x+\varepsilon X\left(H_{2}, H_{3}, x, t\right), \\
H_{2}^{*} & \sim H_{2}+\varepsilon K_{2}\left(H_{2}, H_{3}, x, t\right), \\
H_{3}^{*} & \sim H_{3}+\varepsilon K_{3}\left(H_{2}, H_{3}, x, t\right),
\end{aligned}
$$

which leave equations (1.9)-(1.10) unchanged to order $\varepsilon$, where $\varepsilon \ll 1$. Omitting the details of the derivation, we find the symmetry group of (1.9)-(1.10) has seven parameters and takes the form

$$
\begin{aligned}
T & =\alpha t+c_{t}, \\
X & =\frac{1}{3}(\alpha+m+r) x+c_{x}, \\
\left(\begin{array}{l}
K_{2} \\
K_{3}
\end{array}\right) & =\left(\begin{array}{ll}
m & p \\
q & r
\end{array}\right)\left(\begin{array}{l}
H_{2} \\
H_{3}
\end{array}\right)
\end{aligned}
$$

where $\alpha, m, p, q, r, c_{t}$ and $c_{x}$ are all arbitrary constants. We note that $c_{t}$ and $c_{x}$ represent 

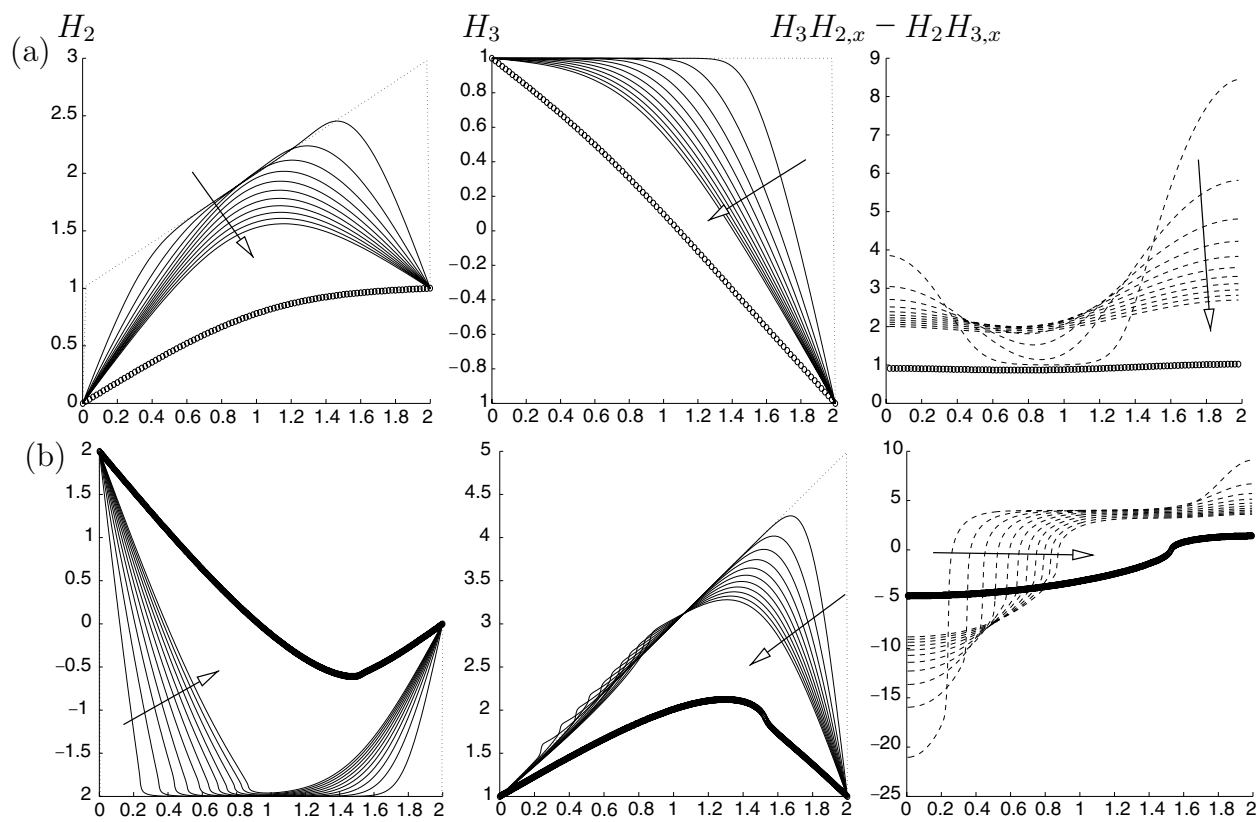

(c)
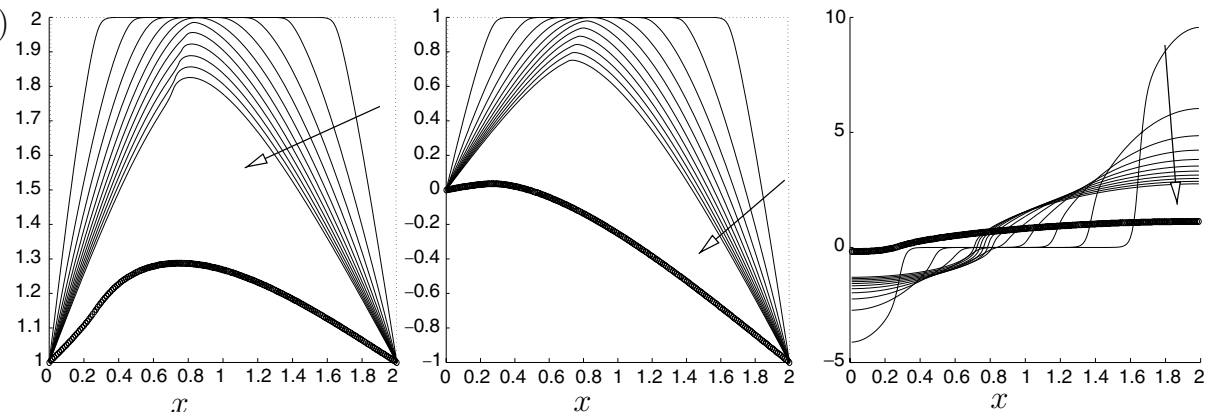

FIGURE 1. (a) Evolution of magnetic fields, following an impulsive change of the boundary magnetic fields, from an initial configuration (dotted line), at uniformly spaced intervals between $t=0.0833$ and $t=0.1$ (solid lines). The final curve (circles) shows the magnetic field at $t=0.5$. (b) An impulsive change of magnetic field from initial configuration $\left(H_{2}, H_{3}\right)=(-2,1+2 x)$ plotted at uniform intervals between $t=0.001667$ and $t=0.02$ (solid lines) the final curve (thick line) is at $t=0.1$. Note that this solution displays a singularity at which $H_{3} H_{2 x}-H_{3} H_{2 x}=0$. (c) An impulsive change to the initially constant magnetic field $\left(H_{2}, H_{3}\right)=(2,1)$. The dotted line shows the initial conditions after that plots are at uniform intervals between $t=0.008333$ and $t=0.1$ (solid lines) and a final plot is made at $t=0.5$ (thick line). Note the presence of two free boundaries propagating in from the edge of the sample.

translations in $t$ and $x ; \alpha$ a rescaling in $t$ and $x$, and $m, p, q$ and $r$ an affine transformation in $\mathrm{H}_{2}$ and $\mathrm{H}_{3}$. In fact, the Lie Group for $\left(\mathrm{H}_{2}, \mathrm{H}_{3}\right)$ is the affine group $\mathrm{A}(2)$.

We can, in the standard way, use the infinitesimal versions of the groups to construct the similarity reductions using the invariant surface condition. Before writing down the the full catalogue of similarity reductions we note that we can simplify its presentation 
by making an appropriate rotation of the $\left(\mathrm{H}_{2}, \mathrm{H}_{3}\right)$ coordinates. The simplified catalogue of reductions then takes the form

$$
\begin{aligned}
& \left.\begin{array}{l}
H_{2}=( \pm t)^{\beta} G(\eta)+K( \pm t)^{\mu} F(\eta) \\
H_{3}=( \pm t)^{\mu} F(\eta) \\
\eta=x( \pm t)^{-(\mu+\beta+1) / 3}
\end{array}\right\} \\
& \left.\begin{array}{l}
H_{2}=\exp (\beta t) G(\eta)+K \exp (\mu t) F(\eta) \\
H_{3}=\exp (\mu t) F(\eta) \\
\eta=x \exp \left(-\frac{\mu+\beta}{3} t\right)
\end{array}\right\} \\
& \left.\begin{array}{l}
H_{2}=K( \pm x)^{3-\beta} F(t)+( \pm x)^{\beta} G(t) \\
H_{3}=( \pm x)^{3-\beta} F(t)
\end{array}\right\} \\
& \left.\begin{array}{l}
H_{2}=( \pm t)^{\mu} G(\eta)+K( \pm t)^{-(1+\mu)} F(\eta) \\
H_{3}=( \pm t)^{-(1+\mu)} F(\eta) \\
\eta=x-p \log ( \pm t)
\end{array}\right\} \\
& H_{2}=\exp (-\mu t) G(\eta)+K \exp (\mu t) F(\eta) \\
& H_{3}=\exp (\mu t) F(\eta) \\
& \eta=x-q t \\
& \left.\begin{array}{l}
H_{2}=\exp (-\mu x) G(t)+K \exp (\mu x) F(t) \\
H_{3}=\exp (\mu x) F(t)
\end{array}\right\} \\
& H_{2}=( \pm t)^{3 \beta} \rho(\eta) \cos (\Omega \log ( \pm t)+\phi(\eta))+L \sin (\Omega \log ( \pm t)+\phi(\eta)) \\
& H_{3}=K( \pm t)^{3 \beta} \rho(\eta) \sin (\Omega \log ( \pm t)+\phi(\eta)) \\
& \eta=x( \pm t)^{-(2 \beta+1 / 3)} \\
& H_{2}=\exp \left(-\frac{3}{2} \mu t\right) \rho(\eta) \cos (\Omega t+\phi(\eta))+L \sin (\Omega t+\phi(\eta)) \\
& H_{3}=\exp \left(-\frac{3}{2} \mu t\right) \rho(\eta) \sin (\Omega t+\phi(\eta)) \\
& \eta=x \exp (\mu t) \\
& \left.\begin{array}{l}
H_{2}=( \pm x)^{3 / 2} \rho(t)[\cos (\Omega \log ( \pm x)+\phi(t))+L \sin (\Omega \log ( \pm x)+\phi(t))] \\
H_{3}=K( \pm x)^{3 / 2} \rho(t) \sin (\Omega \log ( \pm x)+\phi(t))
\end{array}\right\} \\
& H_{2}=( \pm t)^{-1 / 2} \rho(\eta)[\cos (\Omega \log ( \pm t)+\phi(\eta))+L \sin (\Omega \log ( \pm t)+\phi(\eta))] \\
& H_{3}=K( \pm t)^{-1 / 2} \rho(\eta) \sin (\Omega \log ( \pm t)+\phi(\eta)) \\
& \eta=x-q \log ( \pm t)
\end{aligned}
$$




$$
\left.\begin{array}{r}
H_{2}=\rho(x-q t)[\cos (\Omega t+\phi(x-q t))+L \sin (\Omega t+\phi(x-q t))] \\
H_{3}=K \rho(x-q t) \sin (\Omega t+\phi(x-q t)) \\
H_{2}=\rho(t)[\cos (\Omega x+\phi(t))+L \sin (\Omega x+\phi(t))] \\
H_{3}=K \rho(t) \sin (\Omega x+\phi(t)) \\
H_{2}=( \pm t)^{\beta} G(\eta)+K( \pm t)^{\beta} \log ( \pm t) F(\eta) \\
H_{3}=( \pm t)^{\beta} F(\eta) \\
\eta=x( \pm t)^{-(2 \beta+1) / 3} \\
H_{2}=\exp (\beta t)[G(\eta)+q t F(\eta)] \\
H_{3}=\exp (\beta t) F(\eta) \\
\eta=x \exp \left(-\frac{2}{3} \beta t\right) \\
H_{2}=( \pm x)^{3 / 2}(G(t)+q \log ( \pm x) F(t)) \\
H_{3}=( \pm x)^{3 / 2} F(t) \\
H_{2}=( \pm t)^{-1 / 2} G(\eta)+q( \pm t)^{-1 / 2} \log ( \pm t) F(\eta) \\
H_{3}=( \pm t)^{-1 / 2} F(\eta) \\
\eta=x-p \log ( \pm t) \\
H_{2}=( \pm t)^{-1 / 2}(G(x)+q F(x) \log ( \pm t)) \\
H_{3}=( \pm t)^{-1 / 2} F(x) \\
H_{2}=G(\eta)+K t F(\eta) \\
H_{3}=F(\eta)
\end{array}\right\}
$$

where $K, \beta, \mu, p, q, \Omega$ and $L$ are all arbitrary constants. To retrieve the full similarity reductions from this list, one needs simply to make a rotation of the $\left(\mathrm{H}_{2}, \mathrm{H}_{3}\right)$ axes of the form

$$
H_{2} \rightarrow \cos (\chi) H_{2}-\sin (\chi) H_{3}, \quad H_{3} \rightarrow \cos (\chi) H_{3}+\sin (\chi) H_{2},
$$

(where $\chi$ is arbitrary) so that, for instance, (4.4) now becomes

$$
\left.\begin{array}{l}
H_{2}=\cos (\chi)( \pm t)^{\beta} G(\eta)+(K \cos (\chi)-\sin (\chi))( \pm t)^{\mu} F(\eta), \\
H_{3}=(\cos (\chi)+K \sin (\chi))( \pm t)^{\mu} F(\eta)+\sin (\chi)( \pm t)^{\beta} G(\eta), \\
\eta=x( \pm t)^{-(\mu+\beta+1) / 3} .
\end{array}\right\}
$$




\section{Travelling wave solutions}

Where we look for travelling wave solutions to (1.9)-(1.10) of the form

$$
\begin{aligned}
& H_{2}=\cos (\chi) G(x-q t)-\sin (\chi) F(x-q t), \\
& H_{3}=\cos (\chi) F(x-q t)+\sin (\chi) G(x-q t), \\
& \eta=x-q t,
\end{aligned}
$$

(where $K$ and $\chi$ are arbitrary constants) we obtain the following equations for $F$ and $G$ :

$$
\begin{aligned}
& -q F^{\prime}=\frac{d}{d \eta}\left(F^{\prime}\left|F G^{\prime}-G F^{\prime}\right|\right), \\
& -q G^{\prime}=\frac{d}{d \eta}\left(G^{\prime}\left|F G^{\prime}-G F^{\prime}\right|\right) .
\end{aligned}
$$

We also note that

$$
H_{3} H_{2 x}-H_{2} H_{3 x}=\left(F G^{\prime}-G F^{\prime}\right) .
$$

Subtracting (5.1) multiplied by $G$ from (5.2) multiplied by $F$ yields the following equation:

$$
-q\left(F G^{\prime}-G F^{\prime}\right)=\left(F G^{\prime}-G F^{\prime}\right)^{\prime}\left|\left(F G^{\prime}-G F^{\prime}\right)\right|+\left(F G^{\prime}-G F^{\prime}\right)\left|\left(F G^{\prime}-G F^{\prime}\right)\right|^{\prime} .
$$

Assuming that $\left(F G^{\prime}-G F^{\prime}\right) \geqslant 0$, we find

$$
\left(F G^{\prime}-G F^{\prime}\right)=-\frac{q}{2}\left(\eta-\eta_{0}\right) \quad \text { for } \quad\left(\eta_{0}-\eta\right) \operatorname{sgn}(q)>0, \quad \text { or } \quad\left(F G^{\prime}-G F^{\prime}\right)=0,
$$

where $\eta_{0}$ is a constant of integration. Substituting (5.3) back into (5.1) and (5.2) we find that $F$ and $G$ take the form

$$
\begin{aligned}
& \left.\begin{array}{c}
F=A+B\left(\eta-\eta_{0}\right)^{2}, \\
G=\left(\frac{q}{4 B}+\frac{A C}{B}\right)+C\left(\eta-\eta_{0}\right)^{2},
\end{array}\right\} \text { for }\left(\eta_{0}-\eta\right) \operatorname{sgn}(q)>0, \\
& F=A \quad \text { and } \quad G=\left(\frac{q}{4 B}+\frac{A C}{B}\right) \quad \text { for }\left(\eta_{0}-\eta\right) \operatorname{sgn}(q)<0,
\end{aligned}
$$

To find solutions with $\left(F G^{\prime}-G F^{\prime}\right) \leqslant 0$ we replace $q$ by $-q$ everywhere in (5.3) and (5.4)-(5.5), except in the inequalities $\left(\eta_{0}-\eta\right) \operatorname{sgn}(q)>0$ and $\left(\eta_{0}-\eta\right) \operatorname{sgn}(q)<0$.

\section{Similarity solutions describing impulsive changes from a constant magnetic field}

In this section, we look for a similarity solution which describes the short-time evolution of the magnetic field when the boundary field, at $x=0$ say, is changed impulsively. With this in mind we look for solutions to (1.9)-(1.10) of the form

$$
H_{2}=G(\eta), \quad H_{3}=F(\eta), \quad \eta=x t^{-1 / 3},
$$

(i.e. (4.4) with $\beta=\mu=0$ ). Substitution of the above into (1.9)-(1.10) leads to coupled 
ODEs for $F$ and $G$

$$
\begin{aligned}
-\frac{\eta}{3} F^{\prime} & =\frac{d}{d \eta}\left(F^{\prime}\left|F G^{\prime}-G F^{\prime}\right|\right), \\
-\frac{\eta}{3} G^{\prime} & =\frac{d}{d \eta}\left(G^{\prime}\left|F G^{\prime}-G F^{\prime}\right|\right) .
\end{aligned}
$$

Multiplying (6.1) by $G$ and subtracting (6.2) multiplied by $F$ then gives rise to the following equation for the diffusion coefficient $F G^{\prime}-G F^{\prime}$ :

$$
\frac{\eta}{3}\left(F G^{\prime}-G F^{\prime}\right)+\frac{d}{d \eta}\left(\left(F G^{\prime}-G F^{\prime}\right)\left|F G^{\prime}-G F^{\prime}\right|\right)=0
$$

which we can solve to find

$$
\begin{aligned}
& F G^{\prime}-G F^{\prime}= \pm \frac{1}{12}\left(A^{2}-\eta^{2}\right) \text { for } \eta^{2} \leqslant A^{2}, \\
& F G^{\prime}-G F^{\prime}=0 \quad \text { for } \eta^{2} \geqslant A^{2} \text {, }
\end{aligned}
$$

where $A$ is a constant of integration. Substituting the solution for $F G^{\prime}-G F^{\prime}$ (6.3) back into (6.1) and (6.2) and integrating for $F$ and $G$, we find

$$
\begin{gathered}
H_{2}=G=\frac{1}{12}\left(\frac{b_{2}-h_{2}}{\left|h_{3} b_{2}-h_{2} b_{3}\right|}\right)\left(A^{2} \eta-\frac{\eta^{3}}{3}\right)+h_{2} \\
\left.H_{3}=F=\frac{1}{12}\left(\frac{b_{3}-h_{3}}{\left|h_{3} b_{2}-h_{2} b_{3}\right|}\right)\left(A^{2} \eta-\frac{\eta^{3}}{3}\right)+h_{3}\right\} \\
H_{2}=G=b_{2}, \quad H_{3}=F=b_{3}, \quad \text { for } \eta^{2} \leqslant A^{2}, \\
\eta=x t^{-1 / 3}
\end{gathered}
$$

where $A$ is given in terms of the four constants of integration $h_{2}, h_{3}, b_{2}, b_{3}$ by

$$
A^{3}=18\left|h_{3} b_{2}-h_{2} b_{3}\right| \text {. }
$$

The similarity solution (6.4)-(6.7) satisfies the following initial value problem:

$$
\begin{gathered}
H_{2}=b_{2}, \quad H_{3}=b_{3}, \quad \text { for } \quad t=0 \quad x \geqslant 0, \\
H_{2}=h_{2}, \quad H_{3}=h_{3}, \quad \text { on } \quad x=0 \quad t>0,
\end{gathered}
$$

and thus describes the evolution from an initially constant field $\left(H_{2}, H_{3}\right)=\left(b_{2}, b_{3}\right)$ in a half-space, when the magnetic field on the boundary $x=0$ is impulsively changed to $\left(H_{2}, H_{3}\right)=\left(h_{2}, h_{3}\right)$ at time $t=0^{+}$. This similarity solution breaks down when $h_{3} b_{2}-h_{2} b_{3}=$ 0 ; this is because the change to the magnetic field (applied on the boundary) is then in the same direction as the magnetic field within the sample. Since the model is invariant under rotation, this is equivalent to setting $H_{3} \equiv 0$ throughout the evolution, and since this implies the diffusion coefficient $\mathrm{H}_{3} \mathrm{H}_{2 x}-\mathrm{H}_{2} \mathrm{H}_{3 x} \equiv 0$ it is not surprising that no similarity solution then exists. We might, however, expect a similarity solution to exist in the scenario where the initial condition takes the form $H_{2}=a x+b, H_{3}=c x+d$, but 

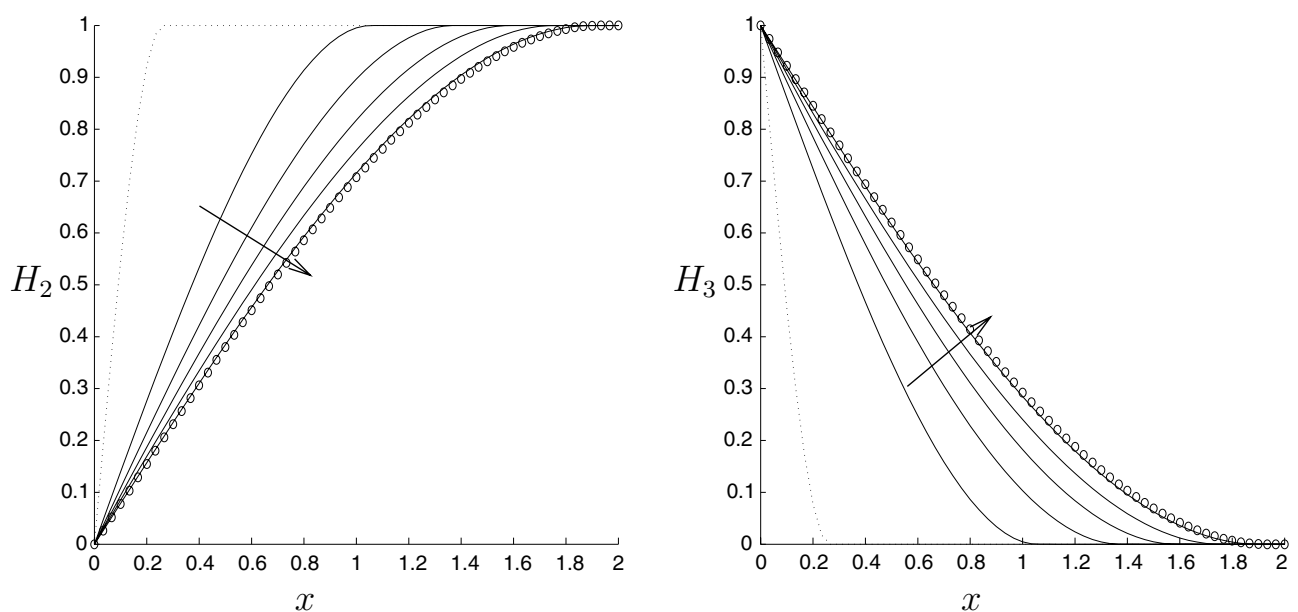

Figure 2. Comparison between numerical solution to (1.9)-(1.10) and the similarity solution (6.4)(6.6). The numerical solution is plotted at equally spaced intervals between times $t=0.01$ and $t=0.4$ (dotted and solid lines). The similarity solution is shown at $t=0.4$ (circles).

for which $h_{3} b-h_{2} d=0$. This motivates the search for another type of similarity solution in $\S 7$.

Numerical evidence suggests that the similarity solution (6.4)-(6.6) provides the short time behaviour of the solution to (1.9)-(1.10) in a slab where an initially constant magnetic field is subjected to an impulsive changes of the boundary magnetic fields. An example of this is provided in Figure 2. This figure shows a numerical solution of (1.9)-(1.10) on the finite domain $0<x<2$. Initial data for the simulation is provided by the similarity solution at $t=0.01$, and the boundary data $H_{2}(0, t)=0, H_{3}(0, t)=1$, $H_{2}(2, t)=1, H_{3}(0, t)=0$ is imposed. The final plot from this simulation is at $t=0.4$ and is compared to the similarity solution (6.4)-(6.6) at the same time (plotted with circles). Note that the similarity solution is exact until the free-boundary $x=A t^{1 / 3}$ reaches the far side of the slab $x=2$. In addition we also might expect this similarity solution to provide the short time behaviour to the equivalent scenario with initial condition provided by the steady solution (2.1). This is exemplified by Figure 3, in which the initial magnetic field in the slab $0<x<2$ is $H_{2}=1+x / 2, H_{3}=1$ and, at time $t=0$, the magnetic field on the left-hand boundary is impulsively changed to $\left.H_{2}\right|_{x=0}=2$, $\left.H_{3}\right|_{x=0}=3$.

Remark 1 This type of solution also describes the evolution of two regions of uniform magnetic field in $x<0$ and $x>0$ which start to interact at time $t=0$. In other words, given the initial conditions

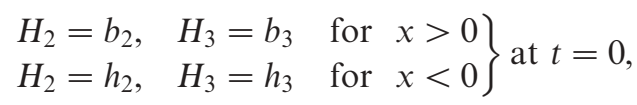



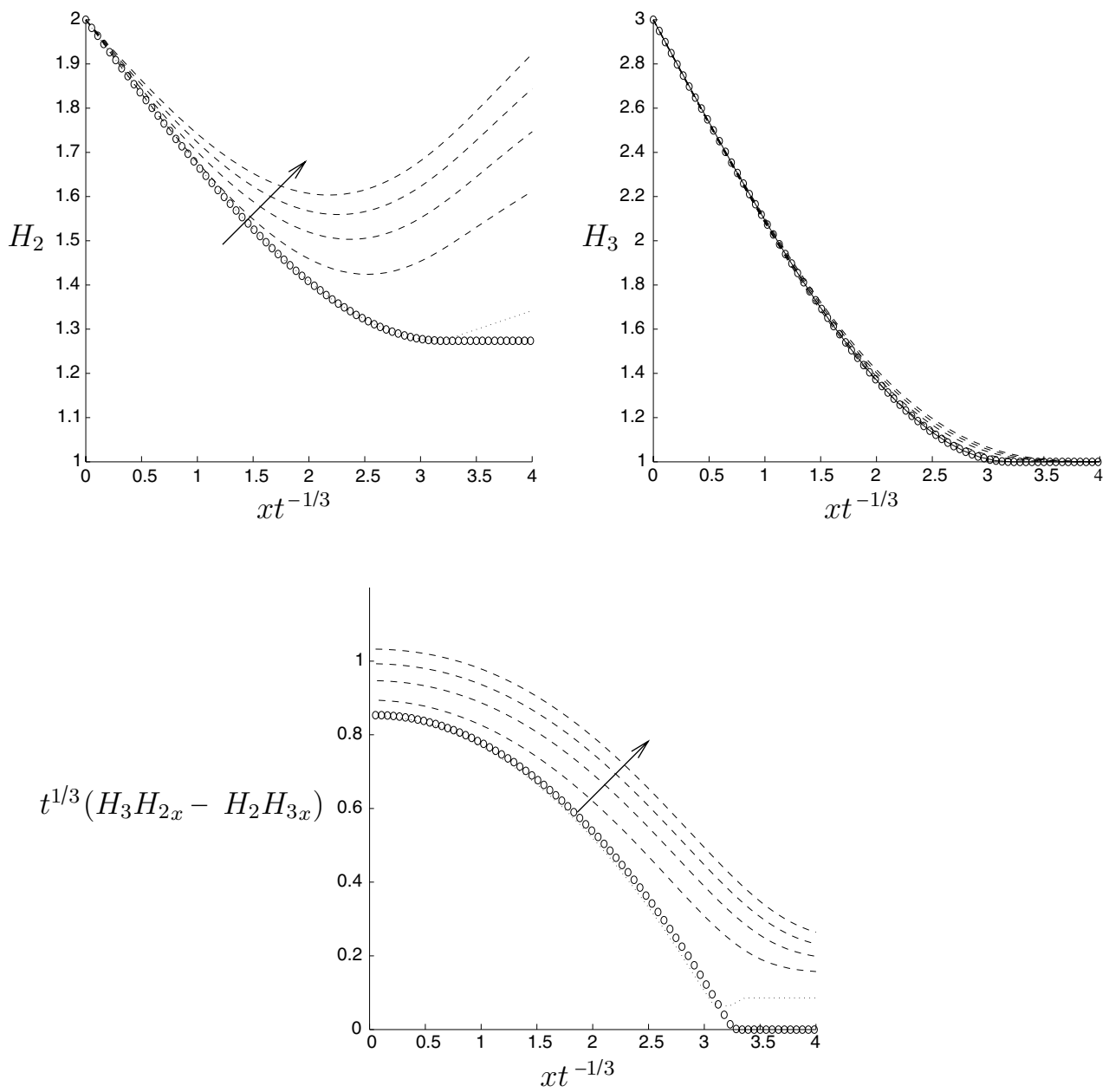

FIGURE 3. A numerical simulation showing the evolution from a steady solution of the form $\mathrm{H}_{2}=\mathrm{ax}+b, \mathrm{H}_{3}=c x+d$ to which an impulsive change of the boundary field at $t=0$ is made. The solution is plotted at equally spaced intervals between times $t=0.005$ and $t=0.1$ (dotted and dashed lines). The similarity solution is shown by circles.

the subsequent evolution is described by

$$
\begin{aligned}
& \left.\begin{array}{l}
H_{2}=G=\frac{\left(b_{2}-h_{2}\right)}{12\left|b_{2} h_{3}-h_{2} b_{3}\right|}\left(A^{2} \eta-\frac{\eta^{3}}{3}\right)+\frac{1}{2}\left(b_{2}+h_{2}\right), \\
H_{3}=F=\frac{\left(b_{3}-h_{3}\right)}{12\left|b_{2} h_{3}-h_{2} b_{3}\right|}\left(A^{2} \eta-\frac{\eta^{3}}{3}\right)+\frac{1}{2}\left(b_{3}+h_{3}\right)
\end{array}\right\} \text { for }|\eta|<A, \\
& H_{2}=G=b_{2} \quad H_{3}=F=b_{3} \\
& \text { for } \eta>A \text {, } \\
& H_{2}=G=h_{2} \quad H_{3}=F=h_{3} \\
& \text { for } \eta<-A \text {, }
\end{aligned}
$$


where

$$
\eta=x t^{-1 / 3}, \quad A^{3}=9\left|b_{2} h_{3}-h_{2} b_{3}\right| .
$$

Remark 2 The similarity reduction $H_{2}=G(\eta), H_{3}=F(\eta), \eta=x(-t)^{-1 / 3}$ is of less physical interest because, as $\eta \rightarrow \infty$ the solutions to $F$ and $G$ are both cubic in $\eta$ (i.e. $F \sim M \eta^{3}$, $\left.G \sim N \eta^{3}\right)$.

\section{Similarity solutions describing an impulsive change to the boundary magnetic field with initial conditions $\mathrm{H}_{2}=a x+b, H_{3}=c x+d$}

As stated in the previous section the similarity solution (6.4)-(6.6), which describes the change from a constant field $\boldsymbol{H}=\left(b_{2}, b_{3}, 0\right)$ when a field $\boldsymbol{H}=\left(h_{2}, h_{3}, 0\right)$ is impulsively applied on the boundary, breaks down when $h_{3} b_{2}-b_{3} h_{2}=0$. This is because the applied field then lies in the same direction as the initial magnetic field. In this section, we look for similarity solutions with initial profile of the form $\mathrm{H}_{2}=a x+b, \mathrm{H}_{3}=c x+d$ to which an impulsive change of magnetic field $\left.\left(H_{2}, H_{3}, 0\right)\right|_{x=0}=\left(h_{2}, h_{3}, 0\right)$ is applied at $t=0^{+}$in the same direction as the field on the boundary so that $h_{2} d-h_{3} b=0$. To do this we look for similarity solutions of the form (4.4) with $\beta=0, \mu=1 / 2$. For simplicity, we write these in their simple forms:

$$
\begin{aligned}
& H_{2}=G\left(x t^{-1 / 2}\right)+K t^{1 / 2} F\left(x t^{-1 / 2}\right), \\
& H_{3}=t^{1 / 2} F\left(x t^{-1 / 2}\right) \\
& \eta=x t^{-1 / 2}
\end{aligned}
$$

or

$$
\begin{aligned}
& H_{2}=G\left(x(-t)^{-1 / 2}\right)+K(-t)^{1 / 2} F\left(x(-t)^{-1 / 2}\right), \\
& H_{3}=(-t)^{1 / 2} F\left(x(-t)^{-1 / 2}\right), \\
& \eta=x(-t)^{-1 / 2}
\end{aligned}
$$

and recall that we can apply an arbitrary rotation of the $\left(H_{2}, H_{3}\right)$-plane to obtain the more general form.

\subsection{Similarity solutions with the form (7.1)}

Substituting (7.1) into (1.9)-(1.10) gives rise to the following equations for $F$ and $G$ :

$$
\begin{aligned}
\left(\frac{1}{2} F-\frac{\eta}{2} F^{\prime}\right) & =\frac{d}{d \eta}\left(F^{\prime}\left|F G^{\prime}-G F^{\prime}\right|\right), \\
-\frac{\eta}{2} G^{\prime} & =\frac{d}{d \eta}\left(G^{\prime}\left|F G^{\prime}-G F^{\prime}\right|\right),
\end{aligned}
$$

which are invariant under three separate discrete transformations

$$
\text { (i) } \eta \rightarrow-\eta, \quad \text { (ii) } F \rightarrow-F, \quad \text { (iii) } G \rightarrow-G \text {. }
$$

We note that

$$
H_{3} H_{2 x}-H_{2} H_{3 x}=F G^{\prime}-G F^{\prime},
$$


and hence that wherever $F G^{\prime}-G F^{\prime}$ vanishes the diffusion coefficient in (1.9)-(1.10) also does so. This system also exhibits two Lie point symmetries and so we could reduce it to a second order non-autonomous system; however we obtain no significant advantage from doing so, and hence choose instead to solve the full system numerically.

Motivated by the initial value problem

$$
\begin{gathered}
H_{2}=m+n x, \quad H_{2}=k x, \quad \text { for } t=0, \\
\left.H_{2}\right|_{x=0}=\beta,\left.\quad H_{3}\right|_{x=0}=0, \quad \text { for } t>0,
\end{gathered}
$$

we look for solutions to (7.3)-(7.4) satisfying the boundary conditions

$$
\begin{array}{cccc}
F=O(\eta), & G \sim \beta & \text { as } & \eta \rightarrow 0, \\
F \sim k \eta, & G \sim m & \text { as } & \eta \rightarrow \infty .
\end{array}
$$

Here $K=n / k$. Assuming that we can find solutions to this boundary value problem, then the long time behaviour of the corresponding solutions to $\mathrm{H}_{2}$ and $\mathrm{H}_{3}$ is given by

$$
\left.\begin{array}{c}
H_{2} \sim \beta+K \alpha x \\
H_{3} \sim k x
\end{array}\right\} \text { as } t \rightarrow \infty
$$

where $\alpha$ is a constant determined by the small $\eta$ behaviour of the solution for $F$ in the following manner:

$$
F \sim \alpha \eta, \quad G \sim \beta+O(\eta) \quad \text { as } \eta \rightarrow 0 .
$$

It is notable that the small $\eta$ behaviour is non-singular, and so exhibits three degrees of freedom.

\subsubsection{Asymptotic behaviour of solutions as $\eta \rightarrow \pm \infty$}

We now look for possible asymptotic behaviours of solutions to (7.3)-(7.4) for large $\eta$. We start by noting that only one exact polynomial solution exists to this system; that is

$$
F=k \eta, \quad G=m,
$$

where $k$ and $m$ are arbitrary constants. In addition to being an exact solution of (7.3)-(7.4) it is possible for other solutions of this system to asymptote to it as $\eta \rightarrow \pm \infty$.

To calculate the number of degrees of freedom exhibited by solutions asymptoting to (7.9), we shall assume that $k m<0$, so that $F G^{\prime}-G F^{\prime}>0$, and perturb about (7.9) as follows:

$$
F \sim k \eta+F_{1}, \quad G \sim m+G_{1}, \quad \eta \rightarrow \pm \infty,
$$

then linearise in $F_{1}$ and $G_{1}$ and seek eigenmodes. This leads to a fourth order linear 
system which we solve to obtain the four eigenmodes

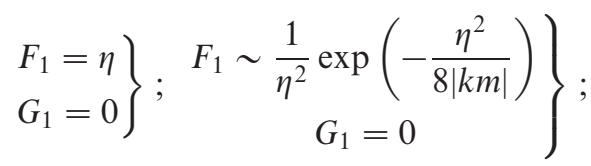

$$
\begin{aligned}
& \left.\left.\begin{array}{ll}
F_{1}=0 \\
G_{1}=1
\end{array}\right\} ; \quad \begin{array}{rl}
F_{1} \sim-\frac{k}{m} \exp \left(-\frac{\eta^{2}}{4|k m|}\right) \\
G_{1} \sim \frac{1}{\eta} \exp \left(-\frac{\eta^{2}}{4|k m|}\right)
\end{array}\right\} .
\end{aligned}
$$

The first and third of these correspond to a small change in $k$ and $m$, respectively, and the second and fourth are both compatible with the asymptotic behaviour (7.9); it follows that this asymptotic behaviour has four degrees of freedom and is thus generic. By the symmetry of the system the same result clearly holds for $\mathrm{km}>0$.

Given that this behaviour exhibits four degrees of freedom and the behaviour at $\eta=0$ exhibits three degrees of freedom we might expect that the boundary value problem comprising equations (7.3)-(7.4) and boundary conditions (7.7) to be well-posed.

\subsubsection{Finite $\eta$ blow-up of solutions to (7.3)-(7.4)}

The system (7.3)-(7.4) exhibits singular behaviour only as $\eta \rightarrow \pm \infty$ or as $F G^{\prime}-G F^{\prime} \rightarrow 0$. The latter leads to solutions with finite $\eta$ blow-up. We have identified two broad classes of such behaviour and believe these to be the only ones. In order to simplify the presentation we write down one example of each class of behaviour noting that we can generate the rest of the class (seven more examples) by application of different combinations of the transformations found in (7.5).

An example of the first class of behaviour is

$$
\left.\begin{array}{c}
F \sim a+b\left(\eta_{0}-\eta\right)^{1 / 2}+\frac{1}{3 a c}\left(\frac{5}{6} \eta_{0} b-b^{2} c\right)\left(\eta_{0}-\eta\right), \\
G-\frac{9 c^{2}}{2} F \sim-c\left(\eta_{0}-\eta\right)^{3 / 2}+O\left(\left(\eta_{0}-\eta\right)^{2}\right) \\
F G^{\prime}-G F^{\prime} \sim \frac{3 a c}{2}\left(\eta_{0}-\eta\right)^{1 / 2}
\end{array}\right\} \text { as } \eta \nearrow \eta_{0}
$$

where $b$ and $\eta_{0}$ are arbitrary constants $a>0$ and $c>0$. Since there are four constants over which we have choice the behaviour is generic (i.e. has four degrees of freedom).

An example of the second class of behaviour is

$$
\left.\begin{array}{c}
F \sim a\left(1-\frac{2}{\eta_{0}}\left(\eta_{0}-\eta\right)-\frac{3}{2 \eta_{0}^{2}}\left(\eta_{0}-\eta\right)^{2} \log \left|\eta_{0}-\eta\right|\right) \\
G \sim-\frac{\eta_{0}}{8 a}\left(\eta_{0}-\eta\right)^{2}-\frac{1}{48 a}\left(\eta_{0}-\eta\right)^{3} \\
F G^{\prime}-G F^{\prime} \sim \frac{\eta_{0}}{4}\left(\eta_{0}-\eta\right)
\end{array}\right\} \text { as } \eta \nearrow \eta_{0}
$$


where $a>0$ and $\eta_{0}>0$ but are otherwise arbitrary. We assess the number of degrees of freedom exhibited by this behaviour by perturbing about it as follows:

$$
\left.\begin{array}{c}
F \sim a\left(1-\frac{2}{\eta_{0}}\left(\eta_{0}-\eta\right)-\frac{3}{2 \eta_{0}}\left(\eta_{0}-\eta\right)^{2} \log \left(\eta_{0}-\eta\right)\right)+F_{1}, \\
G \sim-\frac{\eta_{0}}{8 a}\left(\eta_{0}-\eta\right)^{2}-\frac{1}{48 a}\left(\eta_{0}-\eta\right)^{3}+G_{1}
\end{array}\right\} \text { as } \eta \nearrow \eta_{0}
$$

substituting into (7.1), linearising in $F_{1}$ and $G_{1}$ and seeking eigenmodes to obtain a fourth order linear homogeneous system. The solution to this system, at leading order in $\left(\eta_{0}-\eta\right)$, gives the eigenmodes

$$
\begin{aligned}
& \left.\left.\begin{array}{c}
F_{1} \sim 1-\frac{2}{\eta_{0}}\left(\eta_{0}-\eta\right) \\
G_{1} \sim \frac{\eta_{0}}{8 a^{2}}\left(\eta_{0}-\eta\right)^{2}
\end{array}\right\} ; \quad \begin{array}{c}
F_{1} \sim-\frac{2 a}{\eta_{0}} \\
G_{1} \sim-\frac{\eta_{0}}{4 a}\left(\eta_{0}-\eta\right)
\end{array}\right\} ; \\
& \left.\left.\begin{array}{c}
F_{1} \sim\left(\eta_{0}-\eta\right)^{2} \\
G_{1} \sim O\left(\left(\eta_{0}-\eta\right)^{4}\right)
\end{array}\right\} ; \quad \begin{array}{c}
F_{1} \sim-\frac{4 a^{2}}{\eta_{0}^{3}} \log \left(\eta_{0}-\eta\right) \\
G_{1} \sim 1-\frac{1}{\eta_{0}}\left(\eta_{0}-\eta\right) \log \left(\eta_{0}-\eta\right)
\end{array}\right\} .
\end{aligned}
$$

The first two of these correspond to small changes in $a$ and $\eta_{0}$ respectively; the third is compatible with asymptotic behaviour (7.11) while the fourth is not ( $F_{1}$ being much larger than $F$ in (7.11)). Asymptotic behaviour (7.11) can thus be seen to exhibit three degrees of freedom.

\subsubsection{Solutions with compact support in the diffusion coefficient}

$$
\left|H_{3} H_{2 x}-H_{2} H_{3 x}\right|=\left|F G^{\prime}-G F^{\prime}\right| \text {, and singular solutions }
$$

We can form solutions to (7.3)-(7.4) with compact support in $\left|F G^{\prime}-G F^{\prime}\right|$ by taking a half solution with behaviour (7.11) as $\eta \nearrow \eta_{0}$ (with $\eta_{0}>0$ ) and continuing it by the half solution

$$
F=-k \eta, \quad G=0, \quad F G^{\prime}-G F^{\prime}=0, \quad \eta>\eta_{0}
$$

at $\eta=\eta_{0}$. At the join we must ensure continuity of $F$ and $G$,

$$
[F]_{\eta=\eta_{0}}=0, \quad[G]_{\eta=\eta_{0}}=0,
$$

but since $F G^{\prime}-G F^{\prime}=0$ at the point $\eta=\eta_{0}$ there is no requirement on the continuity of $F^{\prime}$ and $G^{\prime}$. However, we also require continuity of the flux of the fields $\left(\mathrm{H}_{2}, \mathrm{H}_{3}\right)$; this enforces the condition

$$
F^{\prime}\left(F G^{\prime}-G F^{\prime}\right) \rightarrow 0 \quad \text { and } \quad G^{\prime}\left(F G^{\prime}-G F^{\prime}\right) \rightarrow 0 \quad \text { as } \eta \rightarrow \eta_{0} .
$$

Applying these conditions we see that

$$
k=-\frac{a}{\eta_{0}}
$$


We can also form continuous singular solutions to (7.3)-(7.4) by joining the half solution with behaviour (7.10) as $\eta \nearrow \eta_{0}$ to the half solution with behaviour

$$
\left.\begin{array}{c}
F \sim a-b\left(\eta-\eta_{0}\right)^{1 / 2}+\frac{1}{3 a c}\left(\frac{5}{6} \eta_{0} b-b^{2} c\right)\left(\eta-\eta_{0}\right), \\
G-\frac{9 c^{2}}{2} F \sim-c\left(\eta-\eta_{0}\right)^{3 / 2}+O\left(\left(\eta-\eta_{0}\right)^{2}\right) \\
F G^{\prime}-G F^{\prime} \sim \frac{3 a c}{2}\left(\eta-\eta_{0}\right)^{1 / 2}
\end{array}\right\} \text { as } \eta \searrow \eta_{0}
$$

(this behaviour is another member of the class of behaviours to which (7.10) belongs). This ensures that at the point $\eta=\eta_{0}$ there is continuity of $F$ and $G$, and furthermore that the jump conditions

$$
\left[F^{\prime}\left(F G^{\prime}-G F^{\prime}\right)\right]_{\eta=\eta_{0}}=0, \quad\left[G^{\prime}\left(F G^{\prime}-G F^{\prime}\right)\right]_{\eta=\eta_{0}}=0,
$$

are satisfied and hence that there is continuity in the flux of $\mathrm{H}_{2}$ and $\mathrm{H}_{3}$.

Remark We can form other solutions with compact support in $\left|H_{3} H_{2 x}-H_{2} H_{3 x}\right|$, and other singular solutions, by applying any combination of the discrete transformations (7.5) to the solutions we obtain using the methods outlined above.

\subsubsection{Numerical solution of equations (7.3)-(7.4)}

We used a fourth order Runge-Kutta scheme to solve equations (7.3)-(7.4). We found (i) regular solutions, (ii) solutions with compact support in the diffusion coefficient $F G^{\prime}-G F^{\prime}$ and (iii) solutions with an internal singularity. In all cases we required the solution behave like (7.8) at the origin. When searching for solutions to equations (7.3)-(7.4) it is helpful to bear in mind that these equations are invariant under a two parameter group of rescalings

$$
F \rightarrow m F, \quad G \rightarrow \pm \frac{|q|^{3}}{|m|} G, \quad \eta \rightarrow q \eta .
$$

We can use this rescaling invariance to reduce the amount of work needed in searching the solution space. So for example when we look for a solution of the form (i), imposing the condition (7.8) at the origin, it appears that we should obtain a three-parameter family of solutions. However once we have taken account of the invariance of the system to rescalings of the form (7.14) we see that this in fact only a one parameter family of solutions; the other two degrees of freedom merely represent rescalings. Similarly, solutions of the form (ii) have a zero-parameter family of solutions once rescaling have been taken into account and solutions of the form (iii) have a one-parameter family of solutions once rescalings have been accounted for.

An example of (i) which asymptotes to (7.9) as $\eta \rightarrow \infty$ is given in Figure 4(b). An example of (ii) which has behaviour (7.11) as $\eta \nearrow \eta_{0}=3$ and, for $\eta>3$, has form (7.12) (this is shown by dashed lines) is given in Figure 4(a). We also compare this similarity solution to a full numerical solution of the partial differential equations (1.9)-(1.10) in Figure 6. In Figure 5 we show an example of a solution of type (iii), with an internal singularity at $\eta=1$, which asymptotes to (7.9) as $\eta \rightarrow \infty$. Comparison between this 
(a) 0.7

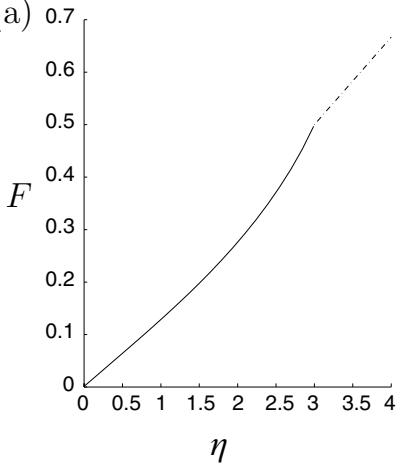

(b)

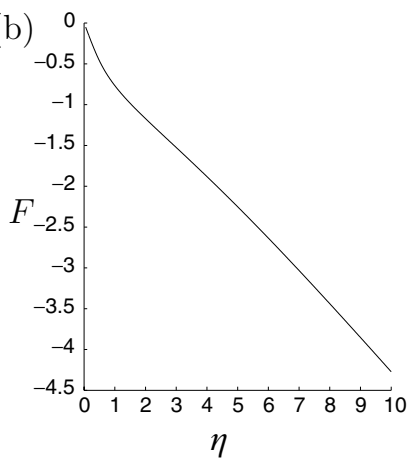

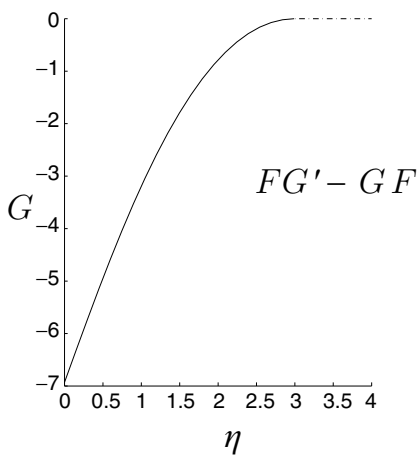
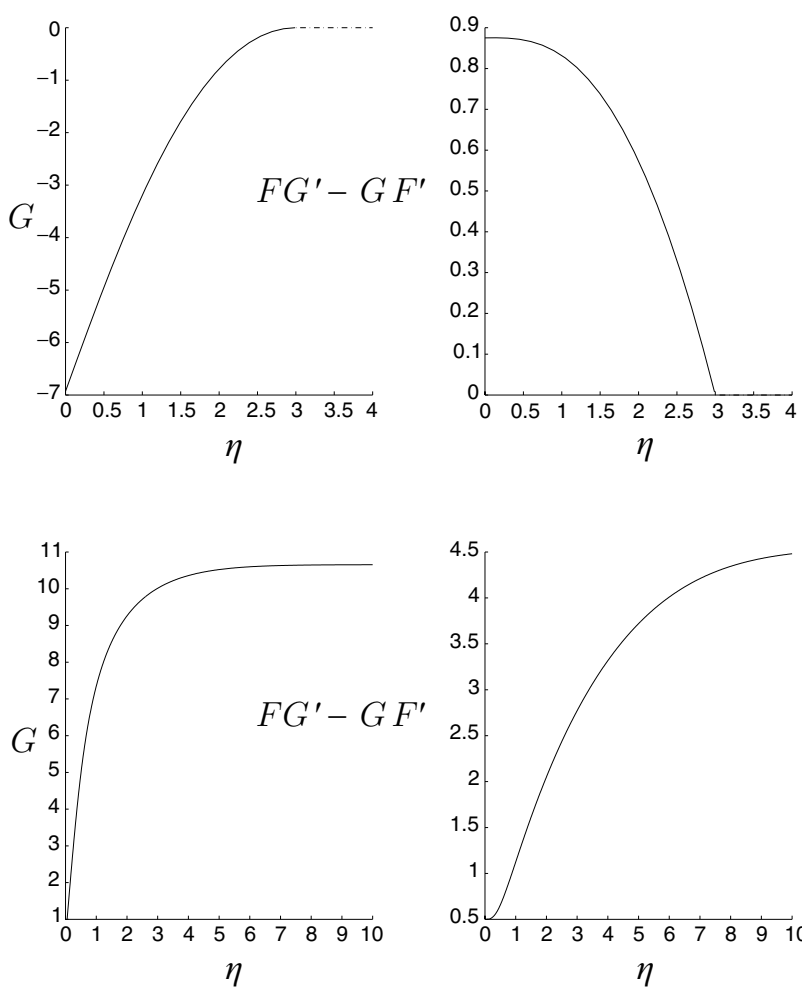

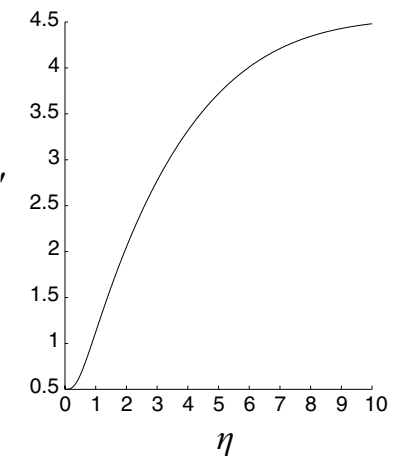

FIGURE 4. Two examples of similarity solution of the form (7.1) with the behaviour (7.8) as $\eta \rightarrow 0$. In (a) we give an example of a solution with compact support which has asymptotic behaviour (7.11) at the point $\eta=\eta_{0}=3$ and which is extended beyond this point by a solution of form (7.12) (the dashed line). In (b) the solution asymptotes to a solution of the form (7.9) as $\eta \rightarrow \infty$.
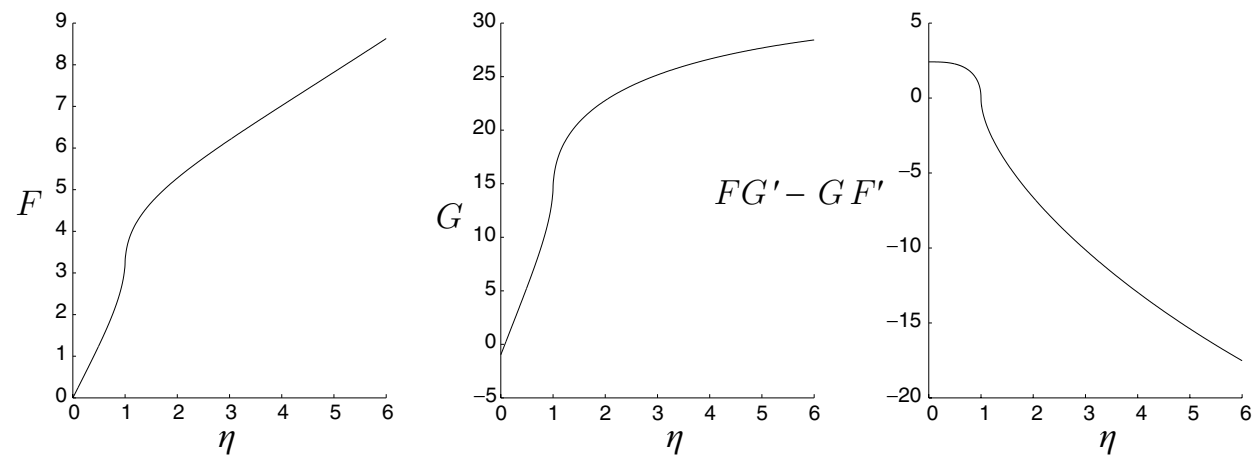

FIGURE 5. This figure shows an example of a similarity solution with singularity at $\eta=1$, with behaviours (7.8) as $\eta \rightarrow 0$ and which asymptotes to (7.9) as $\eta \rightarrow \infty$. It is formed by combining a half solution with asymptotic behaviour (7.10) as $\eta \nearrow 1$ with a half solution with asymptotic behaviour (7.13) as $\eta \searrow 1$. 

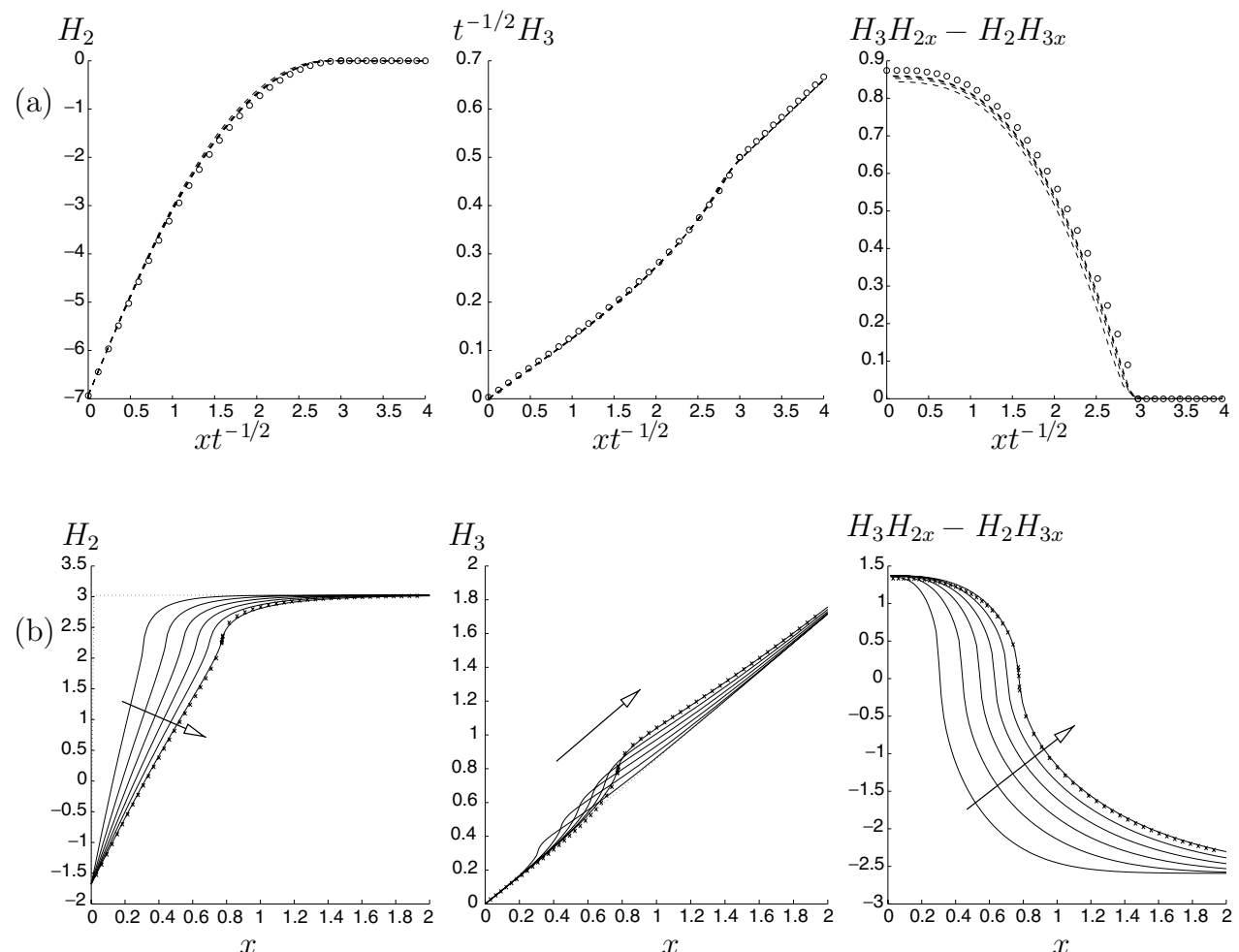

Figure 6. Comparison of numerical solutions to the PDEs with corresponding similarity solution. (a) Comparison to a similarity solution with a free boundary (see Figure 4a):-dashed lines show the numerical solutions plotted at evenly spaced intervals up to $t=0.5$ while circles show the similarity solution at $t=0.5$. (b) Comparison to a similarity solution with a singularity:- solid lines show numerical solution plotted at evenly spaced intervals up to $t=0.15$, dotted line show initial condition, and crosses show the similarity solution at $t=0.15$. In both cases the computational domain is $0 \leqslant x \leqslant 3$.

solution and a solution to the the partial differential equations (1.9)-(1.10) is made in Figure 6.

Numerical work shows that regular solutions (i) to (7.3)-(7.4) which asymptote to (7.8) as $\eta \searrow 0$ and are linear at infinity (7.9) and have the same sign of $F G^{\prime}-G F^{\prime}$ throughout. In contrast to this, singular solutions (iii) to (7.3)-(7.4), which asymptote to (7.8) as $\eta \searrow 0$ and are linear at infinity and have $F G^{\prime}-G F^{\prime}$ which changes sign between the origin and infinity.

\subsubsection{Solutions to (7.3)-(7.4) for which $G$ is a constant}

Solutions to (7.3)-(7.4) exist for which $G$ is constant. Assuming that $F G^{\prime}-G F^{\prime}>0$ and writing

$$
G=-v,
$$


leads to the following second order ODE for $F$ :

$$
\frac{d F}{d \eta}\left(4 v \frac{d^{2} F}{d \eta^{2}}+\eta\right)-F=0, \quad v \frac{d F}{d \eta}>0 .
$$

We consider only the case $v>0$, since this equation is invariant under the discrete transformation

$$
v \rightarrow-v, \quad F \rightarrow-F .
$$

We could now, by rescaling $F$ and $\eta$, scale $v$ out of (7.16). We thus need only consider one value of $v$. We use the fact that this equation is invariant under the transformation

$$
F \rightarrow k^{3} F, \quad \eta \rightarrow k \eta,
$$

to write (7.16) in autonomous form by introducing new variables $\zeta$ and $y$ (an invariant of this transformation) defined by the relations

$$
y=\frac{F}{\eta^{3}}, \quad \zeta=\log |\eta| .
$$

Since (7.16) is invariant under the discrete transformation

$$
F \rightarrow-F, \quad \eta \rightarrow-\eta,
$$

we restrict our attention to solutions for which $\eta>0$. Application of (7.17) to (7.16) then yields the following second order autonomous system

$$
\left.\begin{array}{l}
\frac{d y}{d \zeta}=z, \\
\frac{d z}{d \zeta}=-\frac{1}{4 v}\left(\frac{z+2 y}{z+3 y}\right)-5 z-6 y
\end{array}\right\} \text { where } v(z+3 y) \geqslant 0 \text { and } \eta>0 .
$$

We can describe the nature of solutions to (7.18), and hence to (7.16) also, by sketching the associated phase plane. The null clines of this system are given by

$$
\begin{aligned}
& \frac{d y}{d \zeta}=0 \quad \text { on } \quad z=0 \\
& \frac{d z}{d \zeta}=0 \quad \text { on } \quad z=-\frac{(1+84 v y) \pm\left((36 v y)^{2}+8 v y+1\right)^{1 / 2}}{40 v} \\
& \frac{d z}{d \zeta}= \pm \infty \quad \text { on } \quad z=-3 y,
\end{aligned}
$$

In the region of interest there is a singular point at the origin and no critical points. In Figure 7(b) the null clines of (7.18) are plotted, together with the singular line $y+3 z=0$, for a typical case. The corresponding phase plane is plotted in Figure 7(a).

\section{Asymptotic behaviours of the phase trajectories}

As $\zeta \rightarrow-\infty$ (7.18) the solutions to (7.18) exhibit the following behaviour:

$$
y \sim A \exp (-3 \zeta)+B \exp (-2 \zeta)+\frac{A}{8 v B} \exp (-\zeta) \quad \zeta \rightarrow-\infty,
$$


(a)

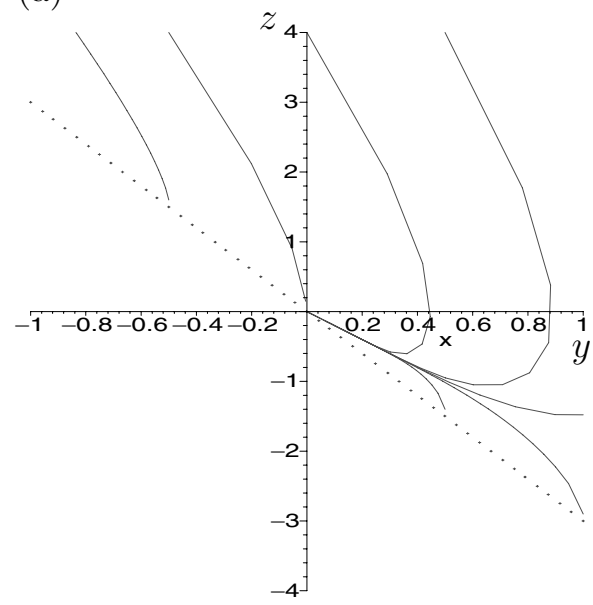

(b)

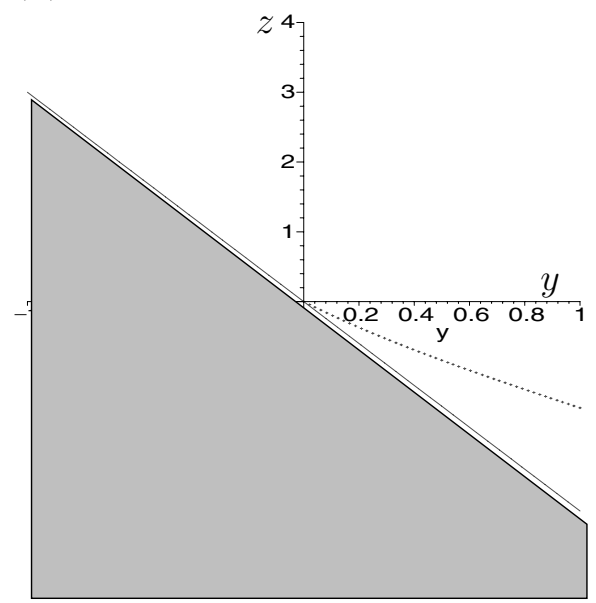

Figure 7. (a) Typical phase paths for the system (7.18) (in this case $v=1 / 20$ ); (b) typical null-clines (dotted lines) and line of singularity (solid line) for the system (the forbidden region of the phase plane is shaded).

where $A$ and $B$ arbitrary; thus $z \sim-3 y$ as $y \rightarrow \infty$ and the corresponding behaviour for $F$ is

$$
F \sim A+B \eta \quad \eta \searrow 0 . \quad(B>0)
$$

As the phase trajectories approach the line $z+3 y=0$ they exhibit either the behaviour

$$
y \sim y_{0}-3 y_{0}\left(\zeta-\zeta_{0}\right)+\frac{1}{3}\left(\frac{2 y_{0}}{v}\right)^{1 / 2}\left(\zeta-\zeta_{0}\right)^{3 / 2} \quad \text { for } y_{0}>0,
$$

or the behaviour

$$
y \sim y_{0}+3 y_{0}\left(\zeta_{0}-\zeta\right)-\frac{1}{3}\left(\frac{-2 y_{0}}{v}\right)^{1 / 2}\left(\zeta_{0}-\zeta\right)^{3 / 2} \quad \text { for } y_{0}<0 .
$$

The corresponding behaviours for $F$ are

$$
F \sim y_{0} \eta_{0}^{3}+\frac{1}{3}\left(\frac{2 y_{0} \eta_{0}^{3}}{v}\right)^{1 / 2}\left(\eta-\eta_{0}\right)^{3 / 2} \quad \text { as } \eta \searrow \eta_{0} \quad\left(y_{0}>0, \quad \eta_{0}>0\right),
$$

and

$$
F \sim y_{0} \eta_{0}^{3}-\frac{1}{3}\left(-\frac{2 y_{0} \eta_{0}^{3}}{v}\right)^{1 / 2}\left(\eta_{0}-\eta\right)^{3 / 2} \text { as } \eta \nearrow \eta_{0} \quad\left(y_{0}<0, \quad \eta_{0}>0\right),
$$

respectively. The latter is equivalent to (7.10) with $b=0$ while the former is equivalent to (7.10) with $b=0$ where we make the transformation $\eta \rightarrow-\eta$ and $G \rightarrow-G$. Finally, we note that trajectories which approach the origin have asymptotic behaviour of the form

$$
y \sim A \exp (-2 \zeta) \quad \zeta \rightarrow \infty
$$


and the corresponding behaviour for $F$ is

$$
F \sim A \eta \quad \eta \rightarrow \infty .
$$

The phase plane reveals that we can find solutions to (7.16) (with positive $v$ ) which link the asymptotic behaviours (7.19) (with $A<0$ ) to (7.21); (7.19) (with $A>0$ ) to (7.22); and (7.20) to (7.22). The only solution to (7.16), which behaves like (7.8) at the origin is the explicit solution $F=B \eta$ (which corresponds to the straight-line solution $z=-2 y$ of (7.18)). It is clear that no solutions exist with compact support in $F G^{\prime}-G F^{\prime} \quad\left(=v F^{\prime}\right)$ and that although we can form singular solutions, as in $\S 7.1 .3$, that these solutions do not exhibit behaviour (7.8) at the origin. The solutions we can find to (7.16) are thus of little physical interest.

\subsubsection{Summary}

The phase plane analysis we have conducted above reveals that the only solutions to (7.3)-(7.4) with $G=$ const. which exhibit local behaviour $F \sim \alpha \eta$ as $\eta \rightarrow 0$ take the form $F=\alpha \eta$. Relaxing the constraint on $G$, and allowing it to be non constant, allows us to find families of solutions to (7.3)-(7.4) which exhibit the behaviour

$$
F \sim \alpha \eta, \quad G \sim \beta+p \eta, \quad \text { as } \quad \eta \rightarrow 0
$$

and which asymptote to a linear solution for large $\eta$

$$
F \sim k \eta, \quad G \sim m \quad \text { as } \eta \rightarrow \pm \infty .
$$

The preceding analysis suggests that, when solving for $F$ and $G$, we should specify three of the five parameters $(\alpha, \beta, p, k, m)$. The motivating initial value problem (7.6) suggests we specify $(\beta, k, m)$. Assuming that we solve only in $\eta>0$ such solutions can either be (i) regular; (ii) have compact support in the diffusion coefficient $F G^{\prime}-G F^{\prime}$ and a corresponding free boundary; or (iii) have an internal singularity. Examples of such solutions are plotted in Figures 4(b), 4(a) and 5 respectively. In Figure 6 comparison is made between numerical solutions calculated from appropriate initial data and two members of this class of similarity solution. Physically such solutions describe the evolution from a steady solution of the form (2.1) in a half-space when an impulsive change of the magnetic field is made on the boundary $x=0$. The evolution is towards another steady solution of the form (2.1). More details on the physical interpretation of such solutions are provided in the conclusion to this work.

\subsection{Similarity solutions with the form (7.2)}

Substituting (7.2) into (1.9)-(1.10) gives the following equations for $F$ and $G$ :

$$
\begin{aligned}
-\left(\frac{1}{2} F-\frac{\eta}{2} F^{\prime}\right) & =\frac{d}{d \eta}\left(F^{\prime}\left|F G^{\prime}-G F^{\prime}\right|\right), \\
\frac{\eta}{2} G^{\prime} & =\frac{d}{d \eta}\left(G^{\prime}\left|F G^{\prime}-G F^{\prime}\right|\right) .
\end{aligned}
$$

We also note that, in this instance,

$$
H_{3} H_{2 x}-H_{2} H_{3 x}=F G^{\prime}-G F^{\prime} .
$$


These equations are again invariant under the discrete transformations (7.5) and we again look for solutions which have behaviour

$$
F \sim \alpha \eta, \quad G \sim \beta+p \eta \quad \text { as } \eta \rightarrow 0,
$$

where $\alpha$ and $\beta$ are arbitrary constants; since such solutions correspond to similarity solutions which evolve from a steady solution of the form $\mathrm{H}_{2}=a x+b, \mathrm{H}_{3}=c x+d$ at $t=-\infty$. The behaviour (7.25) is non-singular and thus clearly has three-degrees of freedom.

\subsubsection{Asymptotic behaviour of solutions as $\eta \rightarrow \pm \infty$}

We note that there are two exact polynomial solutions to (7.23)-(7.24). These are

$$
F=\gamma \eta, \quad G=\beta,
$$

and

$$
F=p \eta^{3} \quad G=-\frac{1}{36 p},
$$

where $p$ is arbitrary. In addition to being exact solutions of (7.23)-(7.24) it is possible for other solutions of this system to asymptote to solutions of these forms as $\eta \rightarrow \pm \infty$. The first of these asymptotic behaviours, namely

$$
F \sim \gamma \eta \quad G \sim \beta \quad \text { as } \eta \rightarrow \pm \infty
$$

exhibits only two degrees of freedom. It follows that this behaviour is always associated with the exact solution (7.26). We calculate the number of degrees of freedom exhibited by solutions asymptoting to (7.27) by perturbing about it

$$
F \sim p \eta^{3}+F_{1}, \quad G \sim-\frac{1}{36 p}+G_{1}, \quad \eta \rightarrow \pm \infty,
$$

linearising in $F_{1}$ and $G_{1}$. This results in a fourth order system for $F_{1}$ and $G_{1}$ whose eigenmodes take the form

$$
\left.\left.\begin{array}{c}
F_{1}=\eta \cos (\sqrt{2} \log \eta) \\
G_{1}=0
\end{array}\right\} ; \quad \begin{array}{c}
F_{1}=\eta \sin (\sqrt{2} \log \eta) \\
G_{1}=0
\end{array}\right\} ;
$$

All of these behaviours, except the last one, are compatible with a solution asymptoting to (7.27); it follows that solutions asymptoting to (7.27) exhibit three degrees of freedom. 
Furthermore, all solutions which display this behaviour at $\infty$ are solutions for which $G$ is constant; such solutions are investigated in the appendix.

\subsubsection{Finite $\eta$ blow-up of the solution}

We can construct the asymptotic behaviours exhibited by solutions to these equations from those found in $\S 7.1$. We identify two possible classes of asymptotic behaviour in which $F G^{\prime}-G F^{\prime} \rightarrow 0$. Examples of these are

$$
\left.\begin{array}{c}
F \sim a+b\left(\eta-\eta_{0}\right)^{1 / 2}+\frac{1}{3 a d}\left(\frac{5}{6} \eta_{0} b-b^{2} d\right)\left(\eta-\eta_{0}\right), \\
G-\frac{9 d^{2}}{2} F \sim d\left(\eta-\eta_{0}\right)^{3 / 2}+O\left(\left(\eta-\eta_{0}\right)^{2}\right) \\
F G^{\prime}-G F^{\prime} \sim \frac{3 a d}{2}\left(\eta-\eta_{0}\right)^{1 / 2}
\end{array}\right\} \text { as } \eta \searrow \eta_{0},
$$

where $b$ and $\eta_{0}$ are arbitrary, and $a$ and $d$ are positive otherwise arbitrary constants; and

$$
\left.\begin{array}{c}
F \sim a\left(1+\frac{2}{\eta_{0}}\left(\eta-\eta_{0}\right)+\frac{3}{2 \eta_{0}^{2}}\left(\eta-\eta_{0}\right)^{2} \log \left|\eta-\eta_{0}\right|\right) \\
G \sim \frac{\eta_{0}}{8 a}\left(\eta-\eta_{0}\right)^{2}-\frac{1}{48 a}\left(\eta-\eta_{0}\right)^{3} \\
F G^{\prime}-G F^{\prime} \sim \frac{\eta_{0}}{4}\left(\eta-\eta_{0}\right)
\end{array}\right\} \begin{gathered}
\text { as } \eta \rightarrow \eta_{0} \\
\left(\eta_{0}\left(\eta-\eta_{0}\right)>0\right)
\end{gathered}
$$

where $a$ is arbitrary and $\eta_{0}>0$ but otherwise arbitrary. The former behaviour (7.28) has four degrees of freedom while the latter exhibits three degrees of freedom. We can construct the other seven members of each class by applying discrete transformations found in (7.5).

Solutions with compact support in $F G^{\prime}-G F^{\prime}$ can be constructed from a half solution in $\eta>\eta_{0}$, which exhibits behaviour (7.29), and joining it to the half solution

$$
F=\frac{a}{\eta_{0}} \eta \quad G=0 \quad \eta \leqslant \eta_{0} .
$$

Singular solutions may be constructed from behaviour (7.28) in a similar manner to that constructed in $\S 7.1 .3$.

\subsubsection{Summary}

The only solutions to (7.23)-(7.24) which display the behaviour

$$
F \sim \gamma \eta \quad G \sim \beta \quad \text { as } \eta \rightarrow \pm \infty,
$$

are the linear solutions $F=\gamma \eta, G=\beta$. The other behaviour which is possible at infinity, namely

$$
F \sim p \eta^{3}, \quad G \sim-\frac{1}{36 p}, \quad \eta \rightarrow \pm \infty
$$


is only exhibited by solutions for which $G$ is a constant. Furthermore none of these solutions have the required behaviour at the origin (i.e. $F \sim \alpha \eta$, and $G \sim \beta$ as $\eta \rightarrow 0$ ). In conclusion, the only solutions to (7.23)-(7.24) which are of direct physical interest are linear ones corresponding to the steady solutions (2.1) of the partial differential equations (1.9)-(1.10).

\section{Similarity solutions which decay to the steady solution (2.2)}

We now look for similarity solutions to (1.9)-(1.10) which decay to one of a degenerate steady solutions of the form (2.2). There are many similarity reductions capable of describing such behaviour (see equations (4.4)-(4.22) for further examples). We choose to look at the similarity reduction (4.4) with $\beta=0, \mu=-1$ which we write in its full form

$$
\left.\begin{array}{l}
H_{2}=\cos \chi G(x)+t^{-1} F(x)(K \cos \chi-\sin \chi) \\
H_{3}=\sin \chi G(x)+t^{-1} F(x)(K \sin \chi+\cos \chi)
\end{array}\right\}
$$

Substitution of the above into (1.9)-(1.10) gives

$$
\begin{aligned}
\frac{d}{d \eta}\left(F^{\prime}\left|F G^{\prime}-G F^{\prime}\right|\right)+F & =0, \\
\frac{d}{d \eta}\left(G^{\prime}\left|F G^{\prime}-G F^{\prime}\right|\right) & =0 .
\end{aligned}
$$

These equations are invariant under the discrete transformations found in (7.5). For convenience, we therefore choose to consider only solutions (or parts of solutions) for which

$$
F G^{\prime}-G F^{\prime} \geqslant 0, \quad \text { and } \quad G^{\prime} \geqslant 0, \quad G \geqslant 0,
$$

noting that we can generate solutions with other combinations of sign for $F G^{\prime}-G F^{\prime}, G^{\prime}$ and $G$ by applying (7.5) to the solutions we find. Integrating (8.3) with respect to $\eta$ gives

$$
F G^{\prime}-G F^{\prime}=\frac{\alpha}{G^{\prime}},
$$

where $\alpha$ is a positive constant of integration. The system given by (8.2) and (8.5) is invariant under translations in $\eta$ as well as being invariant under the Lie point transformation

$$
F \rightarrow e^{4 \varepsilon} F, \quad G \rightarrow e^{-\varepsilon} G, \quad \eta \rightarrow e^{\varepsilon} \eta,
$$

and hence we expect to be able to reduce this third order system to a second order homogeneous system. We use the invariance under translations in $\eta$ by writing

$$
v=\frac{d G}{d \eta}
$$

and hence transforming (8.2) and (8.5) into the system

$$
\alpha v \frac{d^{2} F}{d G^{2}}+F=0, \quad v=\alpha^{1 / 2}\left(F-G \frac{d F}{d G}\right)^{-1 / 2}
$$


(a)

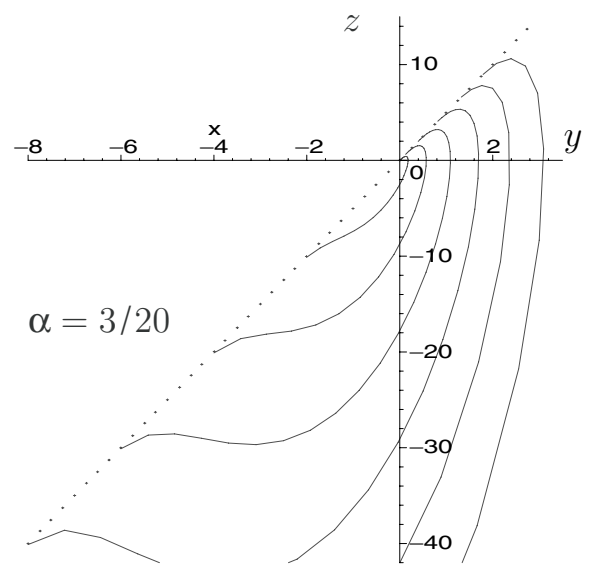

(b)

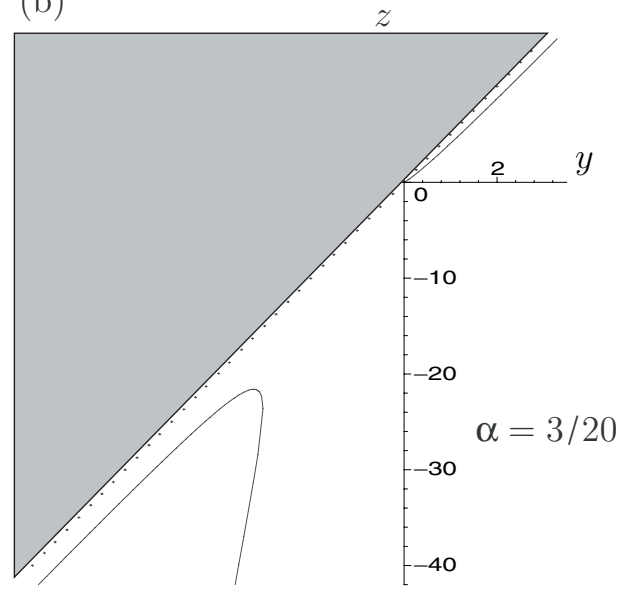

FIGURE 8. (a) Typical phase paths for the system (8.9) (in this case $\alpha=3 / 20$ ); (b) null-clines on which $z^{\prime}=0$ (solid lines). The dotted line marks the edge of the forbidden region $z-5 y<0$ (the forbidden region is shaded).

this system is easily rewritten as a single second order non-autonomous equation for $F$ in terms of $G$. We then introduce two new variables (the first of which is an invariant of the transformation (8.6))

$$
y=F G^{4}, \quad s=\log G,
$$

and rewrite (8.7) as the following second order autonomous system:

$$
\left.\begin{array}{l}
\frac{d y}{d s}=z \\
\frac{d z}{d s}=9 z-20 y-\alpha^{-3 / 2} y(5 y-z)^{1 / 2}
\end{array}\right\} \text { for } 5 y-z>0 .
$$

We study the behaviour of this system by sketching the phase plane and, in order to do this, we consider the null-clines on which $y^{\prime}=0$ and $z^{\prime}=0$. Clearly $y^{\prime}=0$ only on $y=0$. The null-clines corresponding to $z^{\prime}=0$ are given by the curves

$$
z^{ \pm}=\frac{\left(360 y-\alpha^{-3} y^{2}\right) \pm\left(\alpha^{-6} y^{4}+900 \alpha^{-3} y^{3}\right)^{1 / 2}}{162}
$$

and hence in the region $5 y-z>0$

$$
\frac{d z}{d s}=0 \text { on }\left\{\begin{array}{lll}
z=z^{+}(y) & \text { for } & y>0 \\
z=z^{+}(y) & \text { for } & y<-900 \alpha^{3} \\
z=z^{-}(y) & \text { for } & y<-900 \alpha^{3}
\end{array}\right.
$$

These null-clines are plotted, for $\alpha=3 / 20$, in Figure $8(\mathrm{~b})$. There is one critical point at $(y, z)=(0,0)$ about which the solution has asymptotic behaviour

$$
y \sim A e^{5 s}+B e^{4 s}, \quad z \sim 5 A e^{5 s}+4 B e^{4 s}, \quad s \rightarrow-\infty .
$$


The corresponding behaviour for the solution to (8.2) and (8.5) is

$$
\left.\begin{array}{l}
F \sim B+\frac{A \alpha^{1 / 2}}{B^{1 / 2}}\left(\eta-\eta_{0}\right) \\
G \sim \frac{\alpha^{1 / 2}}{B^{1 / 2}}\left(\eta-\eta_{0}\right)-\frac{1}{12 B}\left(\eta-\eta_{0}\right)^{3}
\end{array}\right\} \text { as } \eta \searrow \eta_{0} .
$$

The phase plane is plotted, for $\alpha=3 / 20$, in Figure 8(a). We note that this phase plane is generic in the sense that we can obtain phase planes for other positive values of $\alpha$ simply by rescaling $y$ and $z$ in (8.9). We can see from the phase plane that trajectories can start either at the critical point at the origin or along the line segment $z=5 y$ with $y>0$; they end on the line segment $z=5 y$ with $y<0$. Close to $z=5 y, y$ exhibits the following behaviour

$$
\begin{aligned}
& y \sim y_{0}+5 y_{0}\left(s-s_{0}\right)+\frac{25}{2} y_{0}\left(s-s_{0}\right)^{2}+\left(\frac{125}{6} y_{0}-\frac{y_{0}^{2}}{12 \alpha^{3}}\right)\left(s-s_{0}\right)^{3}, \quad \text { as } s \rightarrow s_{0}, \\
& y_{0}\left(s-s_{0}\right)>0 .
\end{aligned}
$$

The corresponding behaviour for solutions to (8.2) and (8.5) is

$$
\begin{aligned}
& \left.\begin{array}{l}
F \sim \frac{y_{0}}{G_{0}^{4}}+\frac{2 \alpha y_{0}^{1 / 2}}{G_{0}^{7 / 2}}\left(\eta-\eta_{0}\right)^{1 / 2} \\
G \sim G_{0}+\frac{2 \alpha G_{0}^{3 / 2}}{y_{0}^{1 / 2}}\left(\eta-\eta_{0}\right)^{1 / 2}
\end{array}\right\} \text { as } \eta \searrow \eta_{0} \quad \text { for } y_{0}>0 \\
& \left.\begin{array}{rl}
F & \sim \frac{y_{0}}{G_{0}^{4}}+\frac{2 \alpha\left(-y_{0}\right)^{1 / 2}}{G_{0}^{7 / 2}}\left(\eta_{0}-\eta\right)^{1 / 2} \\
G & \sim G_{0}-\frac{2 \alpha G_{0}^{3 / 2}}{\left(-y_{0}\right)^{1 / 2}}\left(\eta_{0}-\eta\right)^{1 / 2}
\end{array}\right\} \text { as } \eta \nearrow \eta_{0} \text { for } y_{0}<0 .
\end{aligned}
$$

The phase plane shows that solutions to (8.2) and (8.5) satisfying (8.4) either link asymptotic behaviours (8.10) to (8.12) or link asymptotic behaviours (8.11) to (8.12).

Consider now the superconducting slab bounded by $x=a$ and $x=b$. Let us look for solutions which describe the scenario in which the magnetic field is held constant on the surfaces of the slab; this corresponds to the condition

$$
F(a)=F(b)=0 .
$$

Looking at the phase plane we see that we can find solutions which start with behaviour (8.10) at some point $\eta=\eta_{0}$ and which, as $\eta$ increases, eventually reach a point at which $F=0$ (i.e. along the negative $z$-axis of the phase plane). The point $\eta=\eta_{0}$ is a regular point of equations (8.2)-(8.3) at which $G=0$. For $\eta<\eta_{0}$ the phase plane argument appears to break down, because $G$ becomes negative. However, by making the transformation $G \rightarrow-G,\left(\eta-\eta_{0}\right) \rightarrow\left(\eta_{0}-\eta\right)$, we see that in fact it still applies and we can thus find solutions to (8.2)-(8.3) for which

$$
F(a)=0, \quad G(a)=G_{a}<0, \quad F(b)=0, \quad G(b)=G_{b}>0, \quad \text { where } a<b .
$$

Examples of such solutions can be found in Figure 9. 

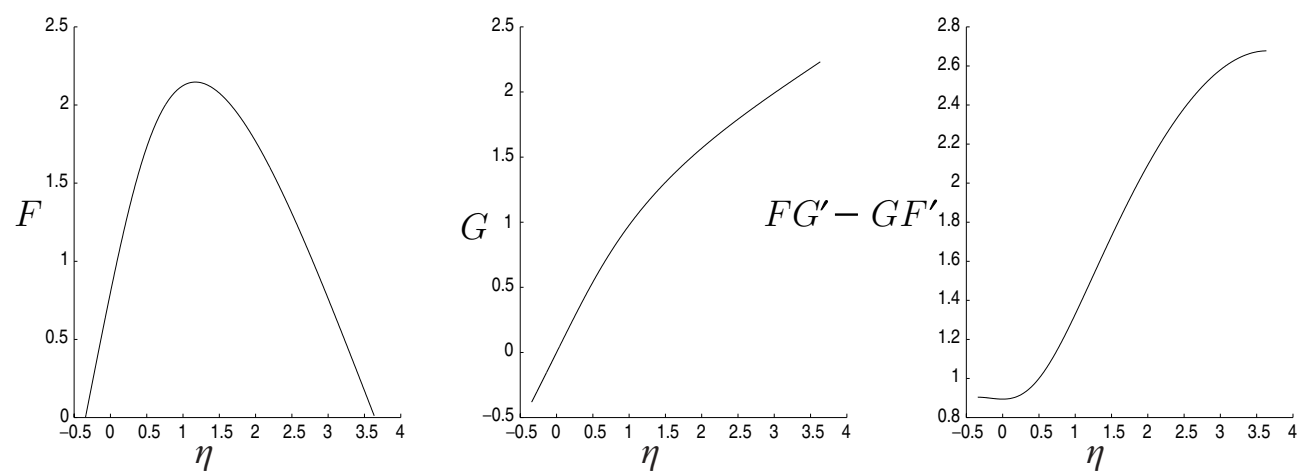

Figure 9. An example of a physically relevant solution to (8.2)-(8.3). Here $\alpha=1$ and $F=0$ on the boundaries of the sample at $a=-0.344$ and $b=3.642$.

\section{Conclusion}

In this work we have investigated a model of superconducting vortex motion in one spatial dimension. We formulated this model in terms of a system of parabolic PDEs (1.7)-(1.8). We noted that this system could be approximated by (1.9)-(1.10), where $H_{3} H_{2 x}-H_{2} H_{3 x} \neq 0$, by neglecting certain small terms. We then investigated this simplified model by first looking for steady solutions. These took either the form

$$
H_{2}=a x+b, \quad H_{3}=c x+d,
$$

or

$$
H_{3}=k H_{2}(x)
$$

The first of these solutions is also an approximate solution to the full model (1.7)-(1.8) whereas the second is not, and in fact evolves over a long-timescale according to a version of the porous medium equation (2.3). We went on to investigate the stability of steady solutions of the form (9.1) and found that all such solutions are stable to small one dimensional perturbations. This, the fact that the equations are parabolic and that the steady solutions are unique for given boundary data, led us to postulate that all solutions to the model with boundary data of the type $\left.\boldsymbol{H}\right|_{x=\alpha}=\boldsymbol{H}^{-},\left.\boldsymbol{H}\right|_{x=\beta}=\boldsymbol{H}^{+}$, where $H_{3}^{+} H_{2}^{-}-H_{3}^{-} H_{2}^{+} \neq 0$, tend to a steady solution of the form $H_{2}=a x+b, H_{3}=c x+d$. Physically this steady state can be interpreted as a state in which $\boldsymbol{j} \cdot \boldsymbol{H}$ is uniform and $\nabla \wedge \boldsymbol{j}=\mathbf{0}$ throughout the sample.

In $\S 3$ we found numerical solutions to the model. These not only supported the the above conjecture but also revealed mathematically interesting behaviour of the time dependent solutions. In particular it is noticeable that solutions exhibit singularities at which the diffusion coefficient goes to zero and that solutions can display compact support in the diffusion coefficient (which leads to a propagating free boundary). Both these behaviours have been noted before in other nonlinear parabolic equations (for example the porous medium equation), but as far as we are aware they have 
not been noted in a system before. In this context we note that Hulshof [15] was the first to find solutions with singularities (at which the diffusion coefficient vanishes) in the porous medium equation, which he termed sign changes, and that the persistence and interaction of these sign changes was subsequently investigated in Hulshof et al. [16].

In $\S 4$ we derived a complete catalogue of similarity reductions to the model. We then used these to look for similarity solutions of physical interest; namely ones which describe the short time behaviour of solutions to the model as either (or both) of the applied magnetic field $\boldsymbol{H}_{\text {appl }}$ and the transport current $\boldsymbol{I}_{\text {trans }}$ are switched on (or off). In $\S 6$ we investigated similarity solutions which describe an impulsive change of the boundary magnetic field applied to an initially constant magnetic field, that is, solutions to the following initial value problem:

$$
\begin{array}{r}
H_{2}=b_{2}, \quad H_{3}=b_{3}, \quad \text { for } \quad t=0 \quad x \geqslant 0, \\
H_{2}=h_{2}, \quad H_{3}=h_{3}, \quad \text { on } \quad x=0 \quad t>0 .
\end{array}
$$

The solutions we found have compact support in the diffusivity, which is associated with a propagating front behind which the diffusivity is nonzero and in front of which it is zero. The similarity reduction breaks down if $b_{2} h_{3}-h_{2} b_{3}=0$ when the change in the magnetic field is in the same direction as the initial field. This phenomenon is essentially a result of the degeneracy of the PDEs and comes about because the initial values take the form of the steady solution $\mathrm{H}_{3}=\mathrm{kH}_{2}(x)$.

This similarity solution can also describe the evolution of two adjacent regions of initially constant magnetic field satisfying the initial value problem

$$
\left.\begin{array}{lll}
H_{2}=b_{2}, & H_{3}=b_{3} & \text { for } x>0 \\
H_{2}=h_{2}, & H_{3}=h_{3} & \text { for } x<0
\end{array}\right\} \text { at } t=0 .
$$

In addition such solutions provide the short time behaviour of an impulsive change to the boundary magnetic field applied to a steady field of the type (9.1). However, in scenarios in which the changes to the boundary field are made in the same direction as the initial field on the boundary, the similarity solution ceases to exist (for the reasons given above). This motivated us to look for another type of similarity solution, in $\S 7$, of the form

$$
\begin{aligned}
& H_{2}=\cos (\chi) G\left(x t^{-1 / 2}\right)+t^{1 / 2}(K \cos (\chi)-\sin (\chi)) F\left(x t^{-1 / 2}\right) \\
& H_{3}=t^{1 / 2}(K \sin (\chi)+\cos (\chi)) F\left(x t^{-1 / 2}\right)+\sin (\chi) G\left(x t^{-1 / 2}\right) \\
& \eta=x t^{-1 / 2}
\end{aligned}
$$

(where $K$ and $\chi$ are arbitrary constants). We were able to find solutions to the following boundary value problem:

$$
\begin{aligned}
& F(\eta)=O(\eta) \quad G(\eta) \sim \beta \quad \text { as } \eta \rightarrow 0, \\
& F(\eta) \sim k \eta \quad G(\eta) \sim m \quad \text { as } \eta \rightarrow \infty,
\end{aligned}
$$


which correspond to the following initial value problem for $\mathrm{H}_{2}$ and $\mathrm{H}_{3}$ :

$$
\left.\begin{array}{l}
H_{2}=m \cos \chi+k x(K \cos \chi-\sin \chi) \\
H_{3}=m \sin \chi+k x(K \sin \chi+\cos \chi)
\end{array}\right\} \text { at } t=0 \quad x>0,
$$

with

$$
\left.\begin{array}{l}
H_{2}=\beta \cos \chi \\
H_{3}=\beta \sin \chi
\end{array}\right\} \text { on } x=0, \quad t>0 .
$$

Note that $\left.\left.H_{2}\right|_{x=0, t>0} H_{3}\right|_{x=0, t=0}-\left.\left.H_{3}\right|_{x=0, t>0} H_{2}\right|_{x=0, t=0}=0$, or in other words that the change in the magnetic field on the boundary is in the same direction as the initial magnetic field on the boundary. The long time behaviour to this initial value problem is

$$
\left.\begin{array}{l}
H_{2} \sim \beta \cos \chi+\alpha x(K \cos \chi-\sin \chi) \\
H_{3} \sim \beta \sin \chi+\alpha x(K \sin \chi+\cos \chi)
\end{array}\right\} \text { as } t \rightarrow \infty,
$$

where $\alpha=\left.(d F / d \eta)\right|_{\eta=0}$.

Our analysis shows that solutions to the ODEs (7.3)-(7.4) are either (i) regular (ii) have compact support in $F G^{\prime}-G F^{\prime}=H_{3} H_{2 x}-H_{2} H_{3 x}$ or (iii) have a singularity at which $F G^{\prime}-G F^{\prime}$ passes through zero. Physically we expect regular solutions (i) if the large time limit of $\mathrm{H}_{3} \mathrm{H}_{2 x}-\mathrm{H}_{2} \mathrm{H}_{3 x}$ has the same sign as $\left.\left(\mathrm{H}_{3} \mathrm{H}_{2 x}-\mathrm{H}_{2} \mathrm{H}_{3 x}\right)\right|_{t=0}$ (i.e. $\operatorname{sign}(\alpha \beta)=$ $\operatorname{sign}(m k))$ and singular solutions (iii) if these two quantities have differing signs (i.e. $\operatorname{sign}(\alpha \beta)=-\operatorname{sign}(m k))$. If $m=0$ then we obtain solutions with compact support in $F G^{\prime}-$ $G F^{\prime}$ (ii). These conjectures are supported by numerical calculations.

Physically, the initial conditions (9.4) represent a steady solution of the form

$$
H_{2}=a x+b, \quad H_{3}=c x+d,
$$

to which a sudden change of the boundary magnetic field (on $x=0$ ) is imposed (for instance a current is switched on), in the same direction as the initial magnetic field on the boundary. The long term behaviour of the subsequent evolution, described by the similarity solution, is towards another steady solution of this form.

Finally, in $\S 8$ we looked for similarity solutions which decay to steady solutions of the form (9.2). As such steady solutions are of less intrinsic interest than solutions of the form (9.1) we limited our search to solutions of the form

$$
\left.\begin{array}{l}
H_{2}=\cos \chi G(x)+t^{-1} F(x)(K \cos \chi-\sin \chi) \\
H_{3}=\sin \chi G(x)+t^{-1} F(x)(K \sin \chi+\cos \chi)
\end{array}\right\}
$$

which satisfy the ordinary differential equations (8.2)-(8.3). Although we could identify solutions in a sensible physical domain (the slab) with fixed boundary, which decay towards (9.2) for long times, these solutions, unsurprisingly, have singular behaviour at $t=0$. 
Appendix: Solutions to (7.23)-(7.24) for which $G=$ const.

Writing $G=-v$ in equations (7.23)-(7.24) gives

$$
\frac{d F}{d \eta}\left(4 v \frac{d^{2} F}{d \eta^{2}}-\eta\right)+F=0, \quad v \frac{d F}{d \eta}>0 .
$$

We need only consider this equation for one particular value of $v$, which we take to be positive, because we can scale $v$ out of (1) by rescaling $F$ and $\eta$ appropriately. This equation can be reduced to a second order autonomous system by introducing the variables

$$
y=\frac{F}{\eta^{3}}, \quad \zeta=\log \eta
$$

where we assume that $\eta>0$, and leads to

$$
\left.\begin{array}{l}
\frac{d y}{d \zeta}=z \\
\frac{d z}{d \zeta}=\frac{1}{4 v}\left(\frac{z+2 y}{z+3 y}\right)-5 z-6 y
\end{array}\right\} \text { where } v(z+3 y)>0 \text { and } \eta>0
$$

The associated null-clines are given by

$$
\begin{aligned}
& \frac{d y}{d \zeta}=0 \quad \text { on } \quad z=0 \\
& \frac{d z}{d \zeta}=0 \quad \text { on } \quad z=\frac{(1-84 v y) \pm\left((36 v y)^{2}-8 v y+1\right)^{1 / 2}}{40 v} \\
& \frac{d z}{d \zeta}= \pm \infty \quad \text { on } \quad z=-3 y
\end{aligned}
$$

and are plotted in Figure $10 \mathrm{~b}$ for a typical case $(v=1 / 20)$. The corresponding phase trajectories are plotted in Figure 10a. There is a stable critical point at $(y, z)=(1 /(36 v), 0)$ and a singular saddle point at the origin. Furthermore, $z=-2 y$ is an exact solution to these equations.

\section{Asymptotic behaviours of phase trajectories}

For large $y$ the solutions exhibit behaviour of the form

$$
y \sim A \exp (-3 \zeta)+B \exp (-2 \zeta)-\frac{A}{8 v B} \exp (-\zeta) \quad \zeta \rightarrow-\infty
$$

and the corresponding behaviour of $F$ is

$$
F \sim A+B \eta-\frac{A}{8 v B} \eta^{2} \quad \eta \rightarrow 0 .
$$


(a)

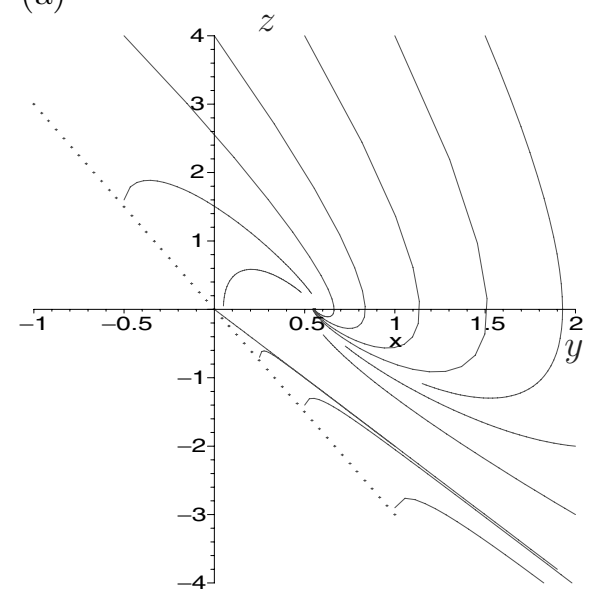

(b)

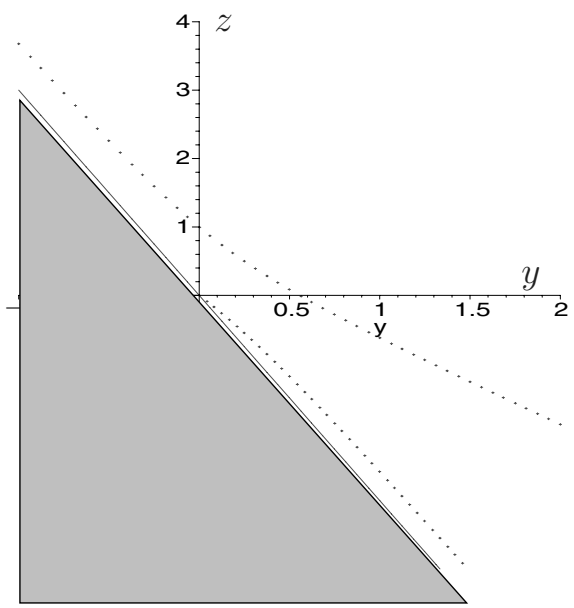

FIgURE 10. (a) Typical phase paths for the system (2) (in this case $v=1 / 20$ ); (b) typical null clines (dotted lines) and line of singularity (solid line) for the system (the forbidden region of the phase plane is shaded).

Phase trajectories close to the line $z+3 y=0$ can either have behaviours of the form

$$
y \sim y_{0}+3 y_{0}\left(\zeta_{0}-\zeta\right)-\frac{1}{3}\left(\frac{2 y_{0}}{v}\right)^{1 / 2}\left(\zeta_{0}-\zeta\right)^{3 / 2} \quad \zeta \nearrow \zeta_{0} \quad\left(y_{0}>0, \quad v>0\right),
$$

or of the form

$$
y \sim y_{0}-3 y_{0}\left(\zeta-\zeta_{0}\right)+\frac{1}{3}\left(\frac{-2 y_{0}}{v}\right)^{1 / 2}\left(\zeta-\zeta_{0}\right)^{3 / 2} \quad \zeta \searrow \zeta_{0} \quad\left(y_{0}<0, \quad v>0\right) .
$$

The corresponding behaviours for $F$ in terms of $\eta$ are

$$
F \sim \eta_{0}^{3} y_{0}-\frac{1}{3}\left(\frac{2 y_{0} \eta_{0}^{3}}{v}\right)^{1 / 2}\left(\eta_{0}-\eta\right)^{3 / 2} \quad \eta \nearrow \eta_{0}>0 \quad\left(y_{0}>0, \quad v>0\right),
$$

and

$$
F \sim \eta_{0}^{3} y_{0}+\frac{1}{3}\left(-\frac{2 y_{0} \eta_{0}^{3}}{v}\right)^{1 / 2}\left(\eta-\eta_{0}\right)^{3 / 2} \quad \eta \searrow \eta_{0}>0 \quad\left(y_{0}<0, \quad v>0\right),
$$

respectively. Trajectories can also approach the critical point $(y, z)=(1 /(36 v), 0)$ as $\zeta \rightarrow \infty$ and this corresponds to $F$ exhibiting the behaviour

$$
F \sim \frac{\eta^{3}}{36 p} \quad \eta \rightarrow \infty
$$

Inspection of the phase-plane reveals that we can find solutions to (1) joining the asymptotic behaviours (3) with $A<0$ to (6); (5) to (6); and (3) with $A>0$ to (4). The 
only solution which behaves like (7.8) at the origin is the explicit solution $F=B \eta$ (which corresponds to the straight-line solution $z=-2 y$ of (7.18)). As in the case of $\S 7.1 .5$, we cannot find non-trivial solutions with compact support and the solutions we can find of this form (i.e $G$ is constant) are of little physical interest.

\section{Acknowledgements}

I would like to acknowledge the support of a Leverhulme Special Research Fellowship and thank Professor J. R. King with whom I had useful discussions about the subject of this work. I would also like to thank an anonymous referee for some useful suggestions.

\section{References}

[1] Abrikosov, A. A. (1957) On the magnetic properties of a superconductor of the second kind. Soviet Phys. J.E.T.P. 5, 174.

[2] Bean, C. P. (1962) Magnetization of hard superconductors. Phys. Rev. Lett. 8, 250-253.

[3] Bluman, G. W. \& Cole, J. D. (1974) Symmetry Methods for Differential Equations. SpringerVerlag.

[4] Bowen, M. \& Witelski, T. P. (2003) Second-type similarity solutions of the thin film equation (in preparation).

[5] Brandt, E. H. (1990) Flux diffusion in high- $T_{c}$ superconductors. Zeit. Phys. B, 80, 165.

[6] Chapman, S. J. (1995) A mean-field model of superconducting vortex motion in threedimensions. SIAM J. Appl. Math. 55, 1259-1274.

[7] Chapman, S. J. (2000) A hierachy of models for type-II superconductors. SIAM Rev. 42 , 555-598.

[8] Chapman, S. J. \& Richardson, G. (1995) Motion of vortices in type-II superconductors. SIAM J. Appl. Math. 55, 1275-1296.

[9] Chapman, S. J. \& Richardson, G. (1999) Personal communication.

[10] Chapman, S. J., Howison, S. D. \& Ockenden, J. R. (1992) Macroscopic models for superconductivity. SIAM Rev. 34(4), 529.

[11] Clem, J. R. (1977) Spiral vortex interaction instability in Type-II superconductors. Phys. Rev. Lett. 38, 1425.

[12] Clem, J. R. \& Perez-Gonzalez, A. (1984) Flux-line-cutting and flux-pinning losses in type-II superconductors in rotating magnetic fields. Phys. Rev. B. 30, 5041-5047.

[13] Du, Q., Gunzburger, M. D. And Peterson, J. S. (1992) Analysis and approximation of the Ginzburg-Landau model of superconductivity. SIAM Rev. 34, 54.

[14] E, W. (1994) Dynamics of vortices in Ginzburg-Landau theories with applications to superconductivity. Physica D, 77 383-404.

[15] Hulshof, J. (1991) Similarity solutions to the porous medium equation with sign changes. J. Math. Anal. Appl. 157, 75-111.

[16] Hulshof, J., King, J. R. \& Bowen, M. (2001) Intermediate asymptotics of the porous medium equation with sign changes. Adv. Diff. Eq. 6, 1115-1152.

[17] Ginzburg, V. L. \& Landau, L. D. (1950) On the theory of superconductivity. J.E.T.P. 20, 1064.

[18] Richardson, G. (1997) Instability of a superconducting line vortex. Physica D, 110, 139-153.

[19] Richardson, G. (2003) Similarity reductions to an averaged model for superconducting vortex motion. Preprint.

[20] Richardson, G. \& Stoth, B. (2000) Ill-posedness of the mean-field model of superconducting vortices and a possible regularisation. Euro. J. Appl. Math. 11, 137-152. 